\title{
AGNs on the Move: A Search for Off-nuclear AGNs from Recoiling Supermassive Black Holes and Ongoing Galaxy Mergers with the Zwicky Transient Facility
}

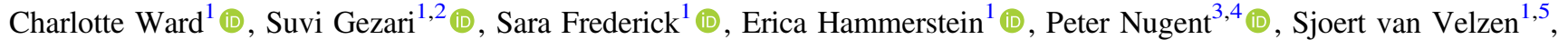 \\ Andrew Drake ${ }^{6}$, Abigail García-Pérez ${ }^{7}$, Immaculate Oyoo ${ }^{8}$, Eric C. Bellm ${ }^{9}$ (iD, Dmitry A. Duev ${ }^{6}$ (i), Matthew J. Graham ${ }^{6}$ (i), \\ Mansi M. Kasliwal ${ }^{6}$ (D), Stephen Kaye ${ }^{10}$, Ashish A. Mahabal ${ }^{6,11}$ (D), Frank J. Masci ${ }^{12}$ (D), Ben Rusholme ${ }^{12}$ (D), \\ Maayane T. Soumagnac ${ }^{3,13}$ (D), and Lin $\operatorname{Yan}^{10}$ (i) \\ ${ }^{1}$ Department of Astronomy, University of Maryland, College Park, MD 20742, USA; charlotteward@astro.umd.edu \\ ${ }_{3}^{2}$ Space Telescope Science Institute, 3700 San Martin Drive, Baltimore, MD 21218, USA \\ ${ }^{3}$ Lawrence Berkeley National Laboratory, 1 Cyclotron Road, Berkeley, CA 94720, USA \\ ${ }^{4}$ Department of Astronomy, University of California, Berkeley, Berkeley, CA 94720, USA \\ ${ }^{5}$ Center for Cosmology and Particle Physics, New York University, NY 10003, USA \\ ${ }^{6}$ Division of Physics, Mathematics, and Astronomy, California Institute of Technology, Pasadena, CA 91125, USA \\ ${ }^{7}$ Instituto Nacional de Astrofísica, Óptica y Electrónica, Tonantzintla, Puebla 72840, México \\ ${ }^{8}$ Prince George's Community College, Largo, MD 20774, USA \\ ${ }^{9}$ DIRAC Institute, Department of Astronomy, University of Washington, 3910 15th Avenue NE, Seattle, WA 98195, USA \\ ${ }^{10}$ Caltech Optical Observatories, California Institute of Technology, Pasadena, CA 91125, USA \\ ${ }^{11}$ Center for Data-Driven Discovery, California Institute of Technology, Pasadena, CA 91125, USA \\ ${ }^{12}$ IPAC, California Institute of Technology, 1200 East California Boulevard, Pasadena, CA 91125, USA \\ ${ }^{13}$ Department of Particle Physics and Astrophysics, Weizmann Institute of Science, Rehovot 76100, Israel \\ Received 2020 November 23; revised 2021 March 24; accepted 2021 March 24; published 2021 May 28
}

\begin{abstract}
A supermassive black hole (SMBH) ejected from the potential well of its host galaxy via gravitational wave recoil carries important information about the mass ratio and spin alignment of the pre-merger SMBH binary. Such a recoiling SMBH may be detectable as an active galactic nucleus (AGN) broad-line region offset by up to $10 \mathrm{kpc}$ from a disturbed host galaxy. We describe a novel methodology using forward modeling with The Tractor to search for such offset AGNs in a sample of 5493 optically variable AGNs detected with the Zwicky Transient Facility (ZTF). We present the discovery of nine AGNs that may be spatially offset from their host galaxies and are candidates for recoiling SMBHs. Five of these offset AGNs exhibit double-peaked broad Balmer lines, which may have arisen from unobscured accretion disk emission, and four show radio emission indicative of a relativistic jet. The fraction of double-peaked emitters in our spatially offset AGN sample is significantly larger than the $16 \%$ double-peaked emitter fraction observed for ZTF AGNs overall. In our sample of variable AGNs we also identified 52 merging galaxies, including a new spectroscopically confirmed dual AGN. Finally, we detected the dramatic rebrightening of SDSS 1133, a previously discovered variable object and recoiling SMBH candidate, in ZTF. The flare was accompanied by the reemergence of strong P Cygni line features, indicating that SDSS 1133 may be an outbursting luminous blue variable star.
\end{abstract}

Unified Astronomy Thesaurus concepts: Active galactic nuclei (16); Galaxy mergers (608); Supermassive black holes (1663)

\section{Introduction}

Supermassive black holes (SMBHs) reside in the center of most galaxies (Kormendy \& Richstone 1995; Ferrarese \& Ford 2005). Galaxy growth via hierarchical mergers therefore results in the formation of SMBH binaries. The time taken for these SMBH binaries to merge depends on the nature of their host galaxies. While binaries in gas-poor galaxies may stall at 1 pc separations (e.g., Milosavljević \& Merritt 2001), SMBH binaries in gas-rich environments may merge on timescales of $10^{6}-10^{7}$ yr (Escala et al. 2005).

A consequence of SMBH mergers in gas-rich environments may be the gravitational wave recoil of coalesced SMBHs after merger. In this process, the asymmetric emission of gravitational waves during SMBH coalescence imparts momentum to the coalesced black hole, ejecting it from the central potential well to wander about the galaxy halo for $10^{6}-10^{9} \mathrm{yr}$ (Volonteri \& Perna 2005; Campanelli et al. 2007; Loeb 2007; Volonteri \& Madau 2008; Blecha \& Loeb 2008). The "recoiling black hole" is expected to carry broad-line gas with it and continue to undergo regulated accretion, allowing it to be observable as an active galactic nucleus (AGN) spatially offset from the center of its host galaxy (Blecha \& Loeb 2008). Simulations by Volonteri \& Madau (2008), for example, show that an AGN with a $500 \mathrm{~km} \mathrm{~s}^{-1}$ kick velocity could still be accreting and be observable as an off-center quasar $30 \mathrm{kpc}$ from its host center.

Other observable signatures of a recoil event include evidence of recent galaxy merging activity. Since the recoiling AGN may continue to accrete for a $10^{6}$ yr timescale after recoil (Blecha \& Loeb 2008), tidal structures may still be visible while the recoiling SMBH is active and detectable. The ejected AGN could also leave behind a trail of feedback evidence in the form of enhanced $\mathrm{H} \alpha$ emission leading to the galaxy center (Loeb 2007).

Simulations show that the recoil velocity and maximum host-AGN spatial offset of the recoiling SMBH depend on the mass ratio and spin alignment of the black hole binary prior to merger (Campanelli et al. 2007; Blecha et al. 2016). Binaries with perfectly aligned spins can produce a maximum recoil velocity of only $200 \mathrm{~km} \mathrm{~s}^{-1}$. A binary with misaligned spins can produce kicks up to $5000 \mathrm{~km} \mathrm{~s}^{-1}$. A population of recoiling 
black holes would therefore provide strong constraints on the distribution of masses and spins in SMBH binaries. This would, in turn, inform simulations of SMBH spin alignment based on torques in the circumbinary gas disk (Bogdanovic et al. 2007; Lodato \& Facchini 2013) and on stellar interactions during inspiral (Berczik et al. 2006).

A confirmed sample of recoiling SMBHs could be used to test predictions of numerical relativity simulations on the fraction of massive black holes ejected via recoil from their host galaxies at different redshifts and the effects of this on observed black hole occupation fractions and the $M_{\mathrm{BH}}-\sigma_{*}$ relation (Volonteri 2007; Volonteri et al. 2010; Blecha et al. 2011). Such a sample would also allow us to study the effect of displaced AGN feedback on the evolution of merger remnants, such as the expected increase in star formation rates and the lengthening of the starburst phase (Blecha et al. 2011).

Despite the many motivations to search for recoiling SMBHs, only a few good candidates have been found to date. One such object is the radio-loud quasi-stellar object (QSO) $3 \mathrm{C}$ 186 , which has an $11 \mathrm{kpc}$ spatial offset from its host galaxy and $\mathrm{a}-2140 \pm 390 \mathrm{~km} \mathrm{~s}^{-1}$ velocity offset between the broad and narrow emission lines (Chiaberge et al. 2017). The tidal features of the host galaxy indicate recent merger activity. Integral field spectroscopy has been performed to study the complex kinematics and determine if the velocity offset could result from a peculiar outflow (Chiaberge et al. 2018). The results are consistent with the recoiling SMBH scenario, but final confirmation will require both James Webb Space Telescope integral field spectroscopy to map the $\mathrm{H} \beta$ region with 0 ." 1 resolution and deep imaging from the Hubble Space Telescope (HST) to rule out the presence of a second, low-mass galaxy (Chiaberge et al. 2018).

SMBH recoil may also be the origin of the variable object SDSS J113323.97+550415.8 (SDSS 1133; Koss et al. 2014). This object is $800 \mathrm{pc}$ from the center of a low-redshift dwarf galaxy and has displayed AGN-like stochastic variability over $>63$ yr. However, AGN-like variability can be mimicked by long-lived stellar transients (Burke et al. 2020) and giant stellar outbursts or supernovae such as SN 2009ip (Maza et al. 2009) and UGC 2773 OT2009-1 (Boles 2009). Spectra of SDSS 1133 show the presence of blueshifted Balmer absorption lines and [Fe II] $\lambda 7155$ and [Ca II] $\lambda \lambda 7291,7324$ forbidden emission lines, which are highly unusual for an AGN. It is therefore possible that SDSS 1133 is a luminous blue variable (LBV) star continuing to demonstrate nonterminal outbursts (Koss et al. 2014).

The lack of many recoiling SMBH candidates has motivated a number of systematic searches for offset AGNs using different techniques. A Gaia analysis of a sample of lowredshift, unobscured broad-line AGNs from the Sloan Digital Sky Survey (SDSS) showed that at least $99 \%$ were within $1 \mathrm{kpc}$ of the host, $90 \%$ were within $500 \mathrm{pc}$, and $40 \%$ were within $100 \mathrm{pc}$ (Shen et al. 2019). That study used a technique called varstrometry (Hwang et al. 2020) to measure AGN-host spatial offsets via the astrometric jitter of the photocenter induced by the AGN flux variability, allowing them to rule out the existence of a substantial offset AGN population on $10 \mathrm{pc}-1$ $\mathrm{kpc}$ scales at redshifts of $0.3<z<0.8$.

From that study, it appears that unobscured, accreting, recoiling SMBHs with $>10$ pc separations must be very rare at low redshifts, if they exist. This may be because SMBH spin alignment is always very efficient, inducing only small velocity recoils with small maximum separations. Recoiling black holes may have also been more common in the early universe due to higher merger rates and lower galaxy masses. Cold gas inflow during mergers may increase the required escape velocity for many galaxies, and gas drag could play a role in keeping recoiling black holes close to the galaxy center (Blecha et al. 2016). The level of accretion may be too small for recoiling SMBHs to be detectable, and offset AGNs may be frequently obscured by the gas environment induced by the merger (Shen et al. 2019).

While these results appear discouraging, another systematic search by Lena et al. (2014) undertook careful isophotal modeling of archival HST images of 14 nearby core elliptical galaxies and found that 10 of the 14 had small $1-10$ pc displacements between the AGN and the host galaxy center, six of which were considered confident detections because the galaxy profiles were not asymmetric. Four of the six galaxies showed alignment between the AGN-photocenter displacements and the radio jet axis. This correlation is predicted for the gravitational recoil of SMBHs but may instead indicate that the spatial offset was induced by the radio jet acceleration of the SMBH. This radio axis correlation would not be produced by interactions with massive perturbers or orbital motion prior to SMBH binary coalescence (Lena et al. 2014).

Other searches have used a multiwavelength approach to successfully find offset AGN candidates. Skipper \& Browne (2018) searched for radio-optical spatial offsets in a sample of 345 SDSS galaxies with nearby compact radio sources detected in the Cosmic Lens All-sky Survey (CLASS) catalog, finding three sources with offsets greater than 0.'6. Condon et al. (2017) found one offset AGN candidate among 492 radio point sources from the NRAO Very Large Array Sky Survey (Condon et al. 1998) when they were crossmatched to extended sources in the Two Micron All Sky Survey's Extended Source Catalog (Jarrett et al. 2000).

A recent study by Reines et al. (2020) found that the majority of a sample of 13 radio AGNs in dwarf galaxies were off-nuclear, likely because the lower escape velocities in dwarf galaxies make it easier for black holes to wander from the central potential. Kim et al. (2017) found a recoiling SMBH candidate in a systematic search for spatially offset X-ray AGNs in a sample of 2542 sources with optical/near-infrared counterparts in archival HST images from the Chandra Source Catalog-SDSS Cross-match Catalog (Evans et al. 2010; Rots \& Budavári 2011).

Although these search strategies have yielded some recoiling SMBH candidates, there are many challenges in confirming the nature of these objects. Candidates with broad-line gas at $>1000 \mathrm{~km} \mathrm{~s}^{-1}$ velocities relative to narrow emission lines but no observable AGN-host spatial offset can often be explained by outflowing winds (Allen et al. 2015; Robinson et al. 2010), scattered broad-line emission from an SMBH binary (Robinson et al. 2010), asymmetric double-peaked emission from an elliptical accretion disk (Steinhardt et al. 2012), or two superposed AGNs (Shields et al. 2009a, 2009b).

For recoiling SMBH candidates with observable host-AGN spatial offsets, it must be ruled out that they are not QSOs with an additional undermassive host (Chiaberge et al. 2017). In this case, the true host galaxy may be on the lower end of the luminosity-SMBH mass scaling relation (McLure \& Dunlop 2002) and may be very compact such that the extended galaxy emission around the QSO is not detectable, resulting in a false 
association with the brighter, offset companion galaxy in a merging system. This can occur when the AGN's host galaxy was tidally stripped as it merged with the larger galaxy (Bellovary et al. 2010), and has been proposed as the nature of the Jonker et al. (2010) recoiling SMBH candidate and the origin of a number of off-nuclear ultraluminous X-ray sources such as HLX-1 (Farrell et al. 2009). Compact dwarf galaxies hosting SMBHs may be very common at low redshifts. Four ultracompact dwarf galaxies with masses $M>10^{7} M_{\odot}$ in the Virgo and Fornax clusters have been shown to host SMBHs through analysis of their velocity dispersion and mass profiles (Seth et al. 2014; Ahn et al. 2017, 2018; Afanasiev et al. 2018). These systems were likely produced through tidal stripping of a larger galaxy hosting an SMBH. Voggel et al. (2019) estimated that such stripped nuclei may host $8 \%-32 \%$ of local SMBHs.

AGNs in merging galaxies also have typical relative velocities of 10-400 $\mathrm{km} \mathrm{s}^{-1}$ (Comerford et al. 2009; Comerford \& Greene 2014; Liu et al. 2018), and these velocities are comparable to the predicted velocities of recoiling SMBHs from spin-aligned binaries. Comerford \& Greene (2014) estimated that $4 \%-8 \%$ of Type II AGNs are in galaxy mergers, so these systems may be quite common. Triple-SMBH systems, in which a merger with a third galaxy occurs before the initial SMBH binary forms, can also be difficult to distinguish from recoiling SMBHs (Civano et al. 2010; Kalfountzou et al. 2017).

Offset AGNs in merging galaxies are nonetheless important to find because they provide a way to study AGN fueling by galaxy-merger-triggered gas inflows (Surace et al. 1998; Canalizo \& Stockton 2001; Treister et al. 2012). The increased incidence of galaxy mergers among X-ray-selected AGNs and the increasing X-ray luminosity with decreasing AGN separation in dual AGNs suggest that black hole accretion peaks during the merging process (Koss et al. 2012). Comerford \& Greene (2014) also found that the fraction of AGNs in galaxy mergers increases from $0.7 \%$ to $6 \%$ over the AGN bolometric luminosity range of $43<\log \left(L_{\mathrm{bol}}\right)\left[\mathrm{erg} \mathrm{s}^{-1}\right]<46$, which suggests that galaxy mergers trigger high-luminosity AGNs. High-resolution hydrodynamical simulations by Van Wassenhove et al. (2012) predicted that AGN triggering and the likelihood of dual-AGN activity are strongest at $<10$ kpc separations and that most merger-triggered AGN activity is nonsimultaneous such that $90 \%$ of SMBHs in mergers appear as single or offset AGNs instead of dual AGNs at $L_{\mathrm{bol}}>10^{44}$ $\mathrm{erg} \mathrm{s}^{-1}$ and at separations of $>1-10 \mathrm{kpc}$. These predictions are supported by observations of the relative occurrence of offset versus dual AGNs (e.g., Comerford et al. 2009). Discoveries of AGNs in tidally stripped dwarf galaxies in mergers may also yield intermediate-mass black hole candidates, which can be used to constrain models of black hole seed formation in the early universe (e.g., Volonteri \& Natarajan 2009; Reines \& Comastri 2016).

Discoveries of AGNs in mergers have occurred both serendipitously and in targeted searches. Binary AGNs were found in X-ray imaging spectroscopy of the ultraluminous infrared galaxy NGC 6240 (Komossa et al. 2003) and of Mrk 739, a galaxy with two optically distinguishable bulges (Koss et al. 2011). A search for AGN companions to a sample of ultrahard X-ray-selected AGNs from the all-sky Swift Burst Alert Telescope survey with Chandra, XRT, and XMM imaging combined with emission line diagnostics with SDSS and Gemini spectroscopy revealed 16 dual AGNs (Koss et al. 2012).

Many searches for AGNs in mergers on $<10 \mathrm{kpc}$ scales have looked for double-peaked narrow [O III] $\lambda 5007$ emission lines in large spectroscopic data sets such as SDSS (e.g., $\mathrm{Xu} \&$ Komossa 2009; Wang et al. 2009; Liu et al. 2010; Smith et al. 2010). Comerford \& Greene (2014) found 351 offset AGN candidates among a sample of 18,314 Type II AGNs by measuring velocity offsets between the forbidden and Balmer emission lines relative to the stellar absorption lines. Fu et al. (2011a) found 16 dual-AGN candidates with high-resolution near-infrared images of 50 double-peaked [O III] $\lambda 5007$ AGNs, and one of these was confirmed as a kiloparsec-scale binary AGN with high-resolution radio images (Fu et al. 2011b).

Searches for dual AGNs via X-ray, radio, and optical imaging suffer from different selection effects, and there are ongoing efforts to understand the effects of AGN obscuration by gas and dust during merger (Koss et al. 2011; Glikman et al. 2015; Kocevski et al. 2015). Koss et al. (2018) found that obscured luminous black holes, with X-ray emission but no visible broad $\mathrm{H} \beta$ lines, are significantly more likely to be in a later-stage nuclear merger than a comparable sample of inactive galaxies.

The lack of a large sample of dual AGNs and even a small sample of confirmed recoiling SMBHs motivates the development of new search strategies to find AGNs offset from their host galaxies and from companion galaxies. A large transient survey that identifies offset AGN candidates via their variability provides one such approach.

In this paper we present a new method for a systematic search for offset AGNs-both recoiling SMBHs and AGNs in galaxy mergers-using the Zwicky Transient Facility (ZTF; Bellm et al. 2019; Graham et al. 2019; Dekany et al. 2020). In Section 2, we present our techniques for filtering ZTF transients to make a sample of 5493 optically variable AGNs. We present a new version of The Tractor forward modeling software for confirmation of AGN-host spatial offsets and describe the candidate selection strategy used to obtain nine offset AGN candidates and 52 AGNs in mergers. In Section 3, we describe the multiwavelength and spectroscopic properties of these new samples and present the rebrightening of the previously discovered recoiling SMBH candidate SDSS 1133.

\section{Sample Selection}

\subsection{The Zwicky Transient Facility}

ZTF is a wide-field optical transient survey of the northern sky that observes in the $g, r$, and $i$ bands with an average 3 day cadence. It uses a 47 square degree field-of-view camera mounted on the Samuel Oschin 48 inch Schmidt telescope at Palomar Observatory. ZTF Phase 1 ran from 2017 November to 2020 October. ZTF identifies variable sources by difference imaging, by which a change in flux is detected by subtracting a high-quality reference image from the data obtained at each epoch. ZTF provides a unique way to search for offset AGNs via their optical variability by giving three main advantages for a systematic AGN search.

First, ZTF provides an opportunity to find new AGNs by applying light-curve modeling techniques to new transients and identifying AGN-like stochastic variability. Light-curve modeling of variable sources to find previously undiscovered AGNs has been demonstrated for SDSS Stripe82 difference imaging 
and for transients in the Palomar Transient Factory (Baldassare et al. 2018, 2020).

Second, image subtractions containing an AGN can be used to locate the position of AGN-like variability relative to the host galaxy. Because spectroscopic surveys tend to have large plate sizes (for example, the SDSS spectroscopic plate size is 1".49), it can be difficult to confirm that the location of the AGN broad lines is associated with an offset point source. By detecting AGN-like variability from an offset point source, we can confirm the spatially offset nature of AGN candidates.

Finally, the sky coverage of ZTF allows us to search a very large area for offset AGNs, which is important given the apparent rarity of $>10 \mathrm{pc}$ spatial offset recoiling SMBHs at low redshifts.

\subsection{Selection of Variable AGNs in ZTF}

We obtain our sample of variable AGNs using data from the ZTF alert stream (Patterson et al. 2019). ZTF alerts contain details of the photometry and astrometry of single-epoch transient detections (Masci et al. 2019). The ZTF pipeline produces approximately 100,000 alerts every night, so we implement a filter with the alert broker and analysis framework AMPEL (Alert Management, Photometry, and Evaluation of Lightcurves; Nordin et al. 2019) to detect variable AGNs among other transient phenomena.

Our method to filter out poor subtractions, moving sources, and variable stars and find only extragalactic transients is similar to the approach used in the tidal disruption event filter of van Velzen et al. (2021). We apply a liberal cut of $<0.8$ on the star-galaxy score (Tachibana \& Miller 2018) to find transients associated with galaxies and a cut of $<0.3$ on the real-bogus score (Duev et al. 2019; Mahabal et al. 2019) to remove bogus transients. We remove objects in busy stellar fields by crossmatching to the Gaia and Pan-STARRS catalogs with catsHTM (Soumagnac \& Ofek 2018) to ensure that there are no more than 30 Gaia objects and 100 Pan-STARRS objects within a $15^{\prime \prime}$ radius. We also require at least three significant detections $>0.01$ days apart and a minimum flux increase of $2.5 \mathrm{mag}$.

This filtering strategy primarily finds two kinds of common extragalactic transients: supernovae and variable AGNs. To select AGNs and remove supernovae within the AMPEL filter, we require that our transients either match an object in a series of AGN catalogs or have variability that is more characteristic of an AGN than of a supernova. We use catsHTM and Extcats ${ }^{14}$ to look for a $2^{\prime \prime}$ crossmatch with objects in the Million Quasars Catalog (Flesch 2015), a machine learningbased catalog of photometric AGN candidates (Brescia et al. 2015), and a catalog of 720,961 variable sources from the Palomar Transient Factory and Intermediate Palomar Transient Factory found between 2009 and 2016 that were not classified as a star (Miller et al. 2017) and had more than five detections over $>24 \mathrm{hr}$.

For sources that do not have an AGN catalog crossmatch, we model their full ZTF light-curve history within the AMPEL filter. We use the SNCOSMO supernova modeling tool (Barbary 2016) to fit the "salt2" SN Ia model to the $g$ - and $r$ band light curves and extract the reduced $\chi_{\mathrm{SN}}^{2}$ goodness of fit for the best-fit SN Ia model.

\footnotetext{
${ }^{14}$ https://github.com/MatteoGiomi/extcats
}

For comparison to the SN Ia goodness of fit, we implement the Butler \& Bloom (2011) quasar modeling routine. This routine calculates the structure function for input light curves and compares this to the ensemble quasar structure function for Sloan Stripe $82 g$ - and $r$-band AGN light curves. The goodness of fit of the ensemble structure function model $\chi_{\mathrm{Q}}^{2}$ gives a measure of how likely the ZTF light curve shows AGN-like variability.

The Butler \& Bloom (2011) routine also calculates the reduced $\chi^{2}$ for the null hypothesis that the source shows nonAGN-like variability (such as from a variable star). We denote this as $\chi_{\mathrm{Q} 0}^{2}$. For the purposes of separating AGNs from supernovae, we find that AGNs generally have much lower $\chi_{\mathrm{Q} 0}^{2}$ values than $\chi_{\mathrm{SN}}^{2}$, so this variability statistic is also effective at separating supernovae from AGNs.

Our filter accepts any source for which either the $g$-band or the $r$-band light curve has a $\chi_{\mathrm{Q}}^{2}$ or $\chi_{\mathrm{Q} 0}^{2}$ value less than the SN Ia goodness of fit $\chi_{\mathrm{SN}}^{2}$. Based on tests with a sample of 111 spectroscopically classified supernovae with $>20$ ZTF epochs, we determine that this method removes approximately $95 \%$ of SN Ia and $60 \%$ of Type II supernovae. With a sample of 166 spectroscopically confirmed AGNs with 20-100 ZTF epochs, we are able to classify $80 \%$ correctly.

Objects that pass either the AGN crossmatch criteria or the light-curve fitting criteria pass the AMPEL filter and are pushed to the GROWTH Marshal science portal for arrangement of spectroscopic follow-up (Kasliwal et al. 2019). The AGN candidates are then confirmed with existing SDSS spectroscopy, with follow-up spectroscopic observations with the DeVeny spectrograph on the Lowell Discovery Telescope (LDT), or by their Wide-field Infrared Survey Explorer (WISE) color or variability history. To classify AGNs based on their WISE W1-W2 color we use the criterion

$$
W 1-W 2>0.662 \exp \left\{0.232(W 2-13.97)^{2}\right\}
$$

from Assef et al. (2013), and to classify AGNs based on significant variability in their WISE light curve we require $\chi^{2}$ relative to a flat light curve to satisfy $\chi^{2} /$ dof $>10$.

We apply this procedure to ZTF alerts from a $2.5 \mathrm{yr}$ period between 2018 January 1 and 2020 July 6 and obtain a sample of 5493 AGNs. This constitutes our final sample of strongly variable ZTF AGNs with spectroscopic or WISE color/ variability confirmation.

\subsection{Selection of AGNs Spatially Offset from Their Host Galaxy}

\subsubsection{Image Modeling with The Tractor}

In order to model the positions of the variable AGNs relative to their host galaxy, we apply The Tractor (Lang et al. 2016) to forward-model the host galaxy profile and transient point-source emission across the ZTF images. The Tractor forward-models in pixel space by parameterizing the astrometry solution, sky noise, and point-spread function of each image and modeling these simultaneously with the shape, flux, and position of each source across images in multiple bands and surveys.

We develop a version of The Tractor to fit a host galaxy profile and an overlapping point source with a position in or around the host. The point-source position and the host galaxy shape, flux, and position are assumed to be constant over all epochs, while the point-source flux may vary across 
single epochs. The version of The Tractor that we apply determines the best-fit one of two galaxy profile models: a pure de Vaucouleurs profile described by $I(r)=I_{0} \exp \left(-7.67\left[\left(r / r_{\mathrm{e}}\right)^{1 / 4}\right]\right)$ and a pure exponential profile described by $I(r)=I_{0} \exp \left(-1.68 r / r_{\mathrm{e}}\right)$. The Tractor is a highly advantageous tool for this analysis because it allows for forward modeling across images of different bands and different instruments. ZTF images taken when the transient is bright can be simultaneously modeled with higher-resolution, deeper images from a different telescope to improve modeling of the host galaxy. Subtraction of the model from the coadded data can also help to reveal irregularities in galaxy structure in the residuals.

We model 3422 out of the sample of 5493 AGNs with The Tractor. Due to limitations with archival storage of ZTF images on our filesystem and the availability of overlapping Legacy Survey (Dey et al. 2019) and Pan-STARRS (Chambers et al. 2016) images for astrometric source matching, we do not model the remaining 2071. As the AGNs are isotropically distributed in the sky, choosing to model a subset of the full sample with The Tractor does not introduce any biases with respect to the distribution of host-AGN offsets.

We remove 126 of the 3422 AGNs because they are duplicates of existing AGNs in the sample. The ZTF alerts for particular transients sometimes demonstrate such a large scatter in position that they are considered to be two or three different transients with separate ZTF names by the alert pipeline. As such, the alerts associated with a single transient can be distributed across two or three different transient objects. By applying an $8^{\prime \prime}$ cone search to all AGNs that pass our AMPEL AGN filter, we find transients that are associated with one another, and select the transient ID with the most alert packets containing real detections.

We model the sample of 3296 unique AGNs by selecting the 30 ZTF $g$ - and $r$-band images taken closest to peak magnitude. This allows us to reach a median depth in $\mathrm{AB}$ magnitude of 22.4 in the $r$ band and of 22.2 in the $g$ band. We choose to model only $30 \mathrm{ZTF}$ images due to computational and time constraints, but future work could model the whole sample with The Tractor to greater depths by modeling larger numbers of ZTF images and including higher-resolution, deeper Dark Energy Camera (DECam) imaging.

Of our 3296 AGNs, 186 do not have sufficient signal-tonoise ratios in the $30 \mathrm{ZTF}$ images to model a host galaxy and point source with The Tractor above the limiting magnitude. This leaves 3110 objects with measured AGN-host offsets.

\subsubsection{Determination of Statistically Significant Spatial Offsets}

Since the ZTF camera has $1^{\prime \prime}$ pixels but much of the simulated recoiling black hole population is only observable at subarcsecond AGN-host spatial offsets, it is important to understand the positional accuracy that can be obtained from ZTF image subtractions.

In order to determine which AGN-host spatial offsets are statistically significant, we study the distribution of offsets from the sample of 3110 objects with host galaxy and point-source positions determined by simultaneously modeling $30 \mathrm{~g}$ - and $r$ band ZTF images with The Tractor (see Section 2.3.1). If the uncertainties in observed R.A. and decl. of the host galaxies are normally distributed with standard deviation $\sigma_{\text {ref }}$ and the uncertainties in observed R.A. and decl. of the variable point sources are normally distributed with standard deviation $\sigma_{\text {sci }}$, the radial distribution of spatial offsets between them will follow a Rayleigh distribution with $\sigma_{\mathrm{R}}^{2}=\sigma_{\text {ref }}^{2}+\sigma_{\text {sci }}^{2}$.

In a study of radio AGNs from the CLASS catalog, Skipper \& Browne (2018) found that the population of offsets between the radio AGNs and optical galaxies in SDSS follow a mixture distribution consisting of a Rayleigh component and an exponential tail component, where the latter component may represent real AGN-host spatial offsets. Similarly, we find that the shape of our offset distribution from Tractor modeling of the ZTF AGN sample is described by the expected Rayleigh distribution at offsets $\lesssim 1^{\prime \prime}$, and at offsets $\gtrsim 1^{\prime \prime}$ the distribution is better described by a decaying exponential.

We therefore model our AGN-host spatial offset distributions by splitting the AGN sample into three different subsamples based on the peak difference magnitude of the AGN. The ranges in peak magnitude used to produce the subsamples are 15-18, 18-19.5, and 19.5-23. These ranges are selected to ensure that each sample is large enough to model the Rayleigh and exponential tail components. The offset distributions of these subsamples are shown in Figures 1(a), (c), and (e). We fit a mixture distribution consisting of a Rayleigh component $\alpha(x)$ and an exponential component $\epsilon(x)$ :

$$
\begin{aligned}
P_{x} & =C \alpha(x)+(1-C) \epsilon(x) \\
& =C \frac{x}{\sigma_{\mathrm{R}}^{2}} \exp \left(-\frac{x^{2}}{2 \sigma_{\mathrm{R}}^{2}}\right)+(1-C) \tau e^{-\tau x}
\end{aligned}
$$

for offset $x$, mixture coefficient $C$, Rayleigh width $\sigma_{\mathrm{R}}$, and exponential decay parameter $\tau$. We do this by directly minimizing the log-likelihood between the model distribution and the data.

We first model the exponential decay parameter $\tau$ to fit only offsets $x>1$ !' 0 so that the fit is not heavily biased by low-offset sources that dominate the distribution. We then fix the value of $\tau$ and fit the mixture distribution to the whole sample to find $\sigma_{\mathrm{R}}$ and $C$. The fits are shown in Figures 1(a), (c), and (e), where we can see the Rayleigh component explaining the portion of the distribution that arises from positional uncertainty and the exponential tail component showing the portion of the distribution that may contain physically real AGN-host offsets. For a given AGN-host offset $x$, we can calculate the probability that an offset $\geqslant x$ is drawn from the Rayleigh component as

$$
P_{\alpha}(x>R)=\frac{C \int_{R}^{\infty} \alpha(x)}{C \int_{R}^{\infty} \alpha(x)+(1-C) \int_{R}^{\infty} \epsilon(x)} .
$$

This is shown as a function of offset $x$ in Figures 1(b), (d), and (f). The probability function shows that as the offset increases, it is less likely to be explained by the Rayleigh distribution arising from positional uncertainties and more likely to be part of an exponential tail consisting of possibly real offsets. The spatial offset at which the probability of being drawn from the exponential component of the mixture distribution is $0.3 \%$ is marked by a dashed line in Figures 1(b), (d), and (f).

Using the probability functions to determine an offset cutoff for the three subsamples, we determine $3 \sigma$ offset cutoffs, shown in the first column of Table 1 . We select a cutoff of 0 ".511 for AGNs with peak magnitudes between 15 and 18, 0 ". 773 for AGNs with peak magnitudes between $18^{\prime \prime}$ and 19". 5, 


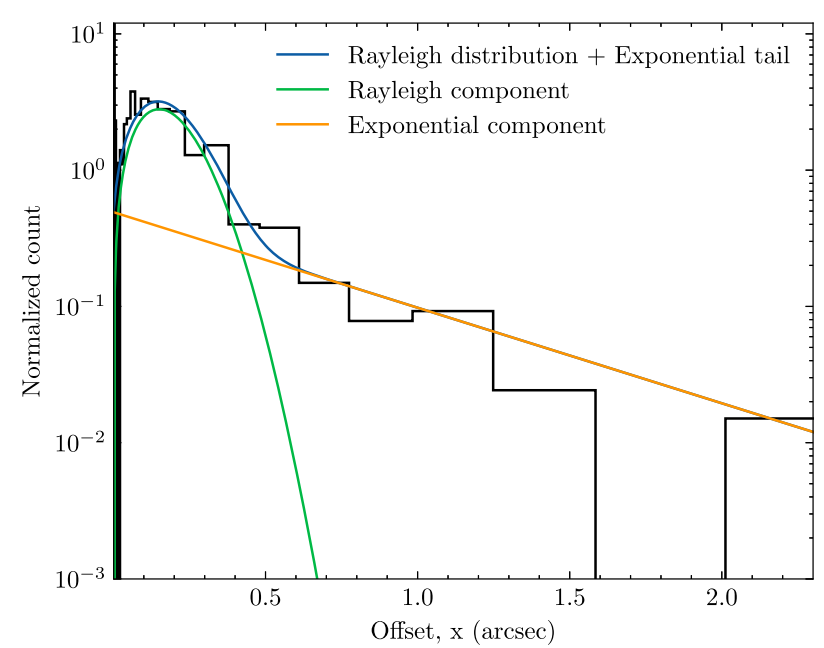

(a) AGN with a peak difference magnitude between 15 and 18 .

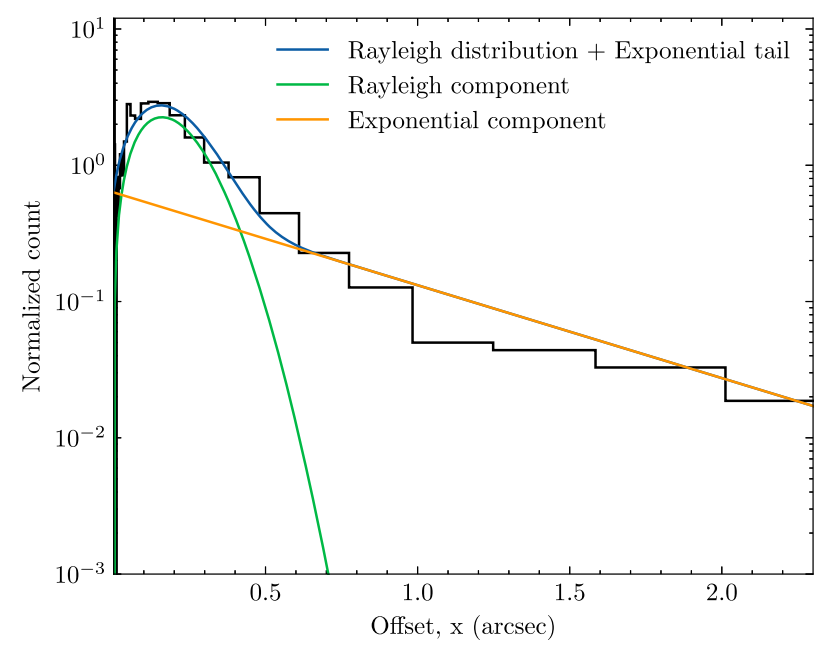

(c) AGN with a peak difference magnitude between 18 and 19.5 .

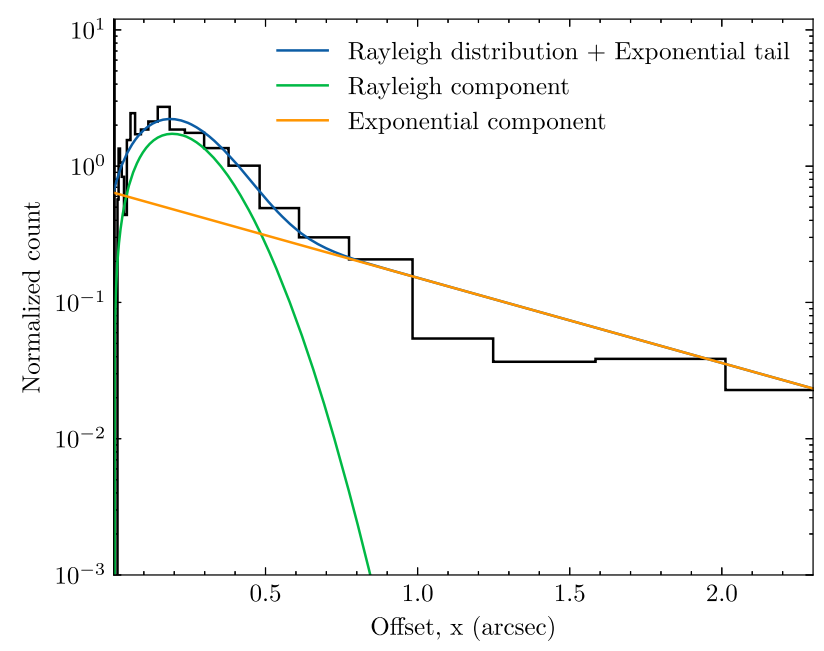

(e) AGN with a peak difference magnitude between 19.5 and 23.

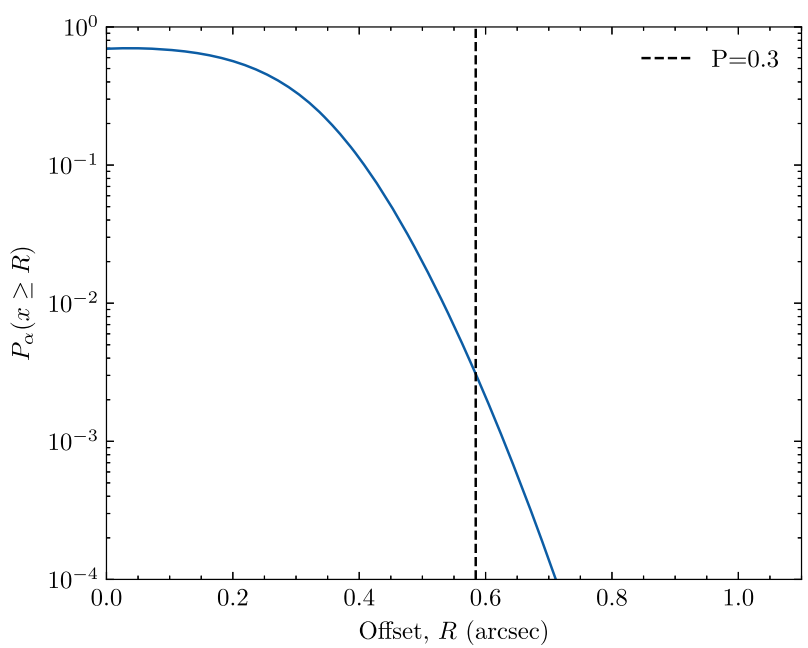

(b) $3 \sigma$ offset $=0.574 "$.

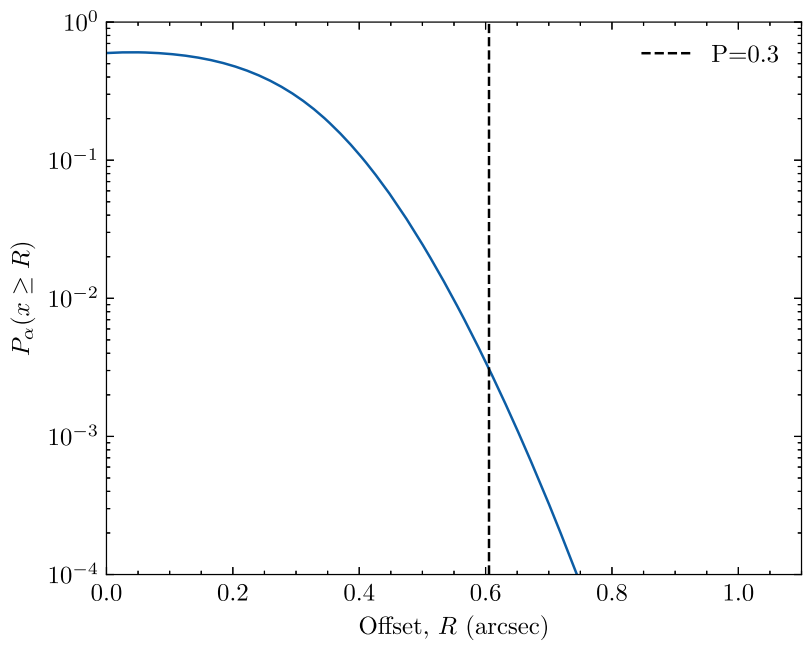

(d) $3 \sigma$ offset $=0.605 "$.

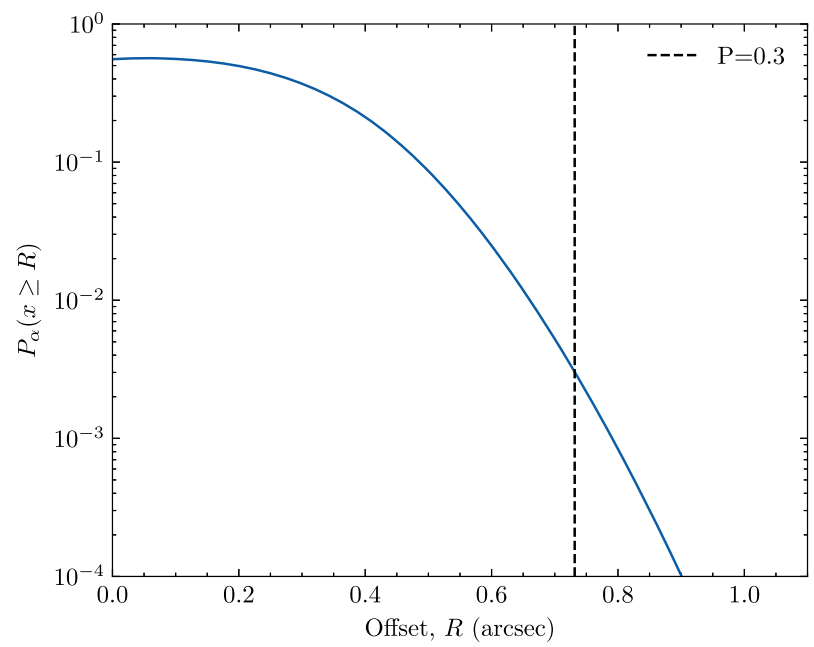

(f) $3 \sigma$ offset $=0.732 "$.

Figure 1. Left: Normalized histogram with logarithmic bins for AGN-host offsets obtained from Tractor modeling. The best-fit model of a mixture distribution with Rayleigh and exponential components is shown. Right: Probability that an offset greater than $R$ is drawn from the Rayleigh component of the mixture distribution shown in (a) instead of the exponential component. The offset where this probability is $0.3 \%$ is shown with a dashed line. 
Table 1

Selected Offset Cutoffs

\begin{tabular}{ccccc}
\hline \hline $\begin{array}{c}\text { Peak } \\
\text { Magnitude }\end{array}$ & $\begin{array}{c}\text { Tractor } \\
\text { Modeling }\end{array}$ & $\begin{array}{c}g \text {-band } \\
\text { Subtraction }\end{array}$ & $\begin{array}{c}r \text {-band } \\
\text { Subtraction }\end{array}$ & Number of AGN \\
\hline $19.5-23$ & 0.732 & 0.946 & 0.976 & 64 \\
$18-19.5$ & 0.605 & 1.009 & 0.773 & 164 \\
$15-18$ & 0.574 & 0.959 & 0.551 & 23 \\
\hline
\end{tabular}

Note. Offset cutoffs (arcseconds) selected for AGNs based on their peak magnitude. The first column shows the peak magnitude bin. The second column shows the $>3 \sigma$ cutoff for a significant AGN-host offset derived by The Tractor. The third and fourth columns show the uncertainty on the magnitude-weighted transient position derived from ZTF alert packets for the $r$ band and $g$ band, respectively. The fifth column shows the number of AGNs that have $>3 \sigma$ Tractor offsets and match the magnitude-weighted transient position using these cutoffs.

and 0".976 for AGNs with peak magnitudes between 19 and 23.

\subsubsection{Matching of Transient Positions from The Tractor and ZTF Difference Images}

In order to confirm that the best-fit point-source position from The Tractor modeling is consistent with the position of the transient in the ZTF difference images, we calculate the magnitude-weighted position of the transient from the ZTF alert packets containing information about the position and magnitude of each single-epoch difference image detection. The weights $1 / \sigma_{\text {offset }}^{2}$ for the magnitude-weighted transient position are calculated using Equation (2) from van Velzen et al. (2019):

$$
\sigma_{\text {offset }}=0.24+0.04\left(m_{\text {diff }}-20\right) .
$$

In order to determine the uncertainty in the magnitudeweighted transient position from the ZTF alert packets, we undertake the same offset distribution modeling procedure as we do for the Tractor AGN-host offsets. The modeling results for each magnitude-binned subsample are shown in Figures 15 and 16 in the Appendix. As the distribution of magnitude-weighted offsets is substantially different for $g$-band images and $r$-band images, due to the differing contributions of the AGN toward the reference image in different bands, we find cutoffs for the two bands separately. Using the probability functions to determine an offset cutoff for the three subsamples, we determine the $3 \sigma$ offset cutoffs shown in Table 1. When checking that the Tractor point-source positions are consistent with the magnitude-weighted transient positions from the alert packets, we require a match within these selected cutoffs.

Figure 2 shows an example ZTF image subtraction for ZTF18aaxvmpg with the Tractor model overlaid. For this object, the Tractor point-source position shown within red contours is consistent with the ZTF transient seen in the image subtraction. This object is therefore considered to be an offset AGN candidate.

We find that 251 AGNs have $>3 \sigma$ AGN-host offsets by Tractor modeling, which are consistent with the magnitudeweighted transient positions from the alert packets. The breakdown of candidates from each peak magnitude bin is shown in the last column of Table 1.

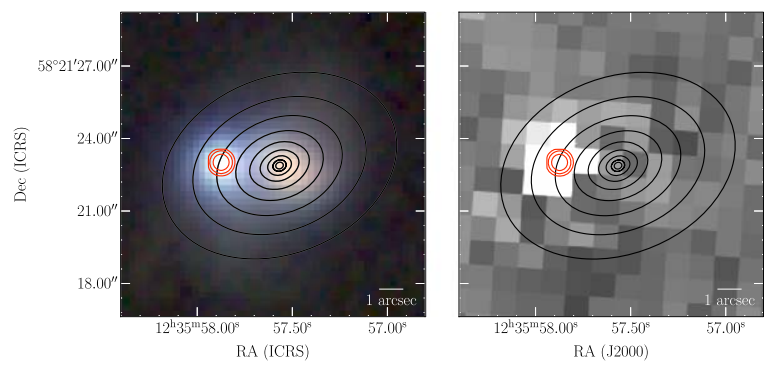

Figure 2. Left: Coadded $g-, r$-, and $z$-band Legacy Survey images of ZTF18aaxvmpg. Right: ZTF image subtraction of ZTF18aaxvmpg when the AGN was close to peak magnitude. Overlaid contours show the best-fit Tractor galaxy profile (black) and point-source model (red) for a theoretical seeing of $1^{\prime \prime}$ derived from ZTF image modeling.

Table 2

Classification of Offset AGNs

\begin{tabular}{lc}
\hline \hline Classification & Number \\
\hline AGNs in galaxy mergers & 52 \\
AGNs offset from the stellar bulge of a disturbed galaxy & 9 \\
AGNs aligned with the stellar bulge of a disturbed galaxy & 21 \\
AGNs offset from an undisturbed galaxy & 29 \\
AGNs without position confirmation in Legacy Survey modeling & 140 \\
\hline Total & 251
\end{tabular}

Note. The breakdown of the complete sample of 251 offset AGNs into five morphology-based classifications. The first row is the number of AGNs that have extended galaxy emission around them and appear to be interacting and merging with a second galaxy. The second row shows the number of AGNs that are not surrounded by a stellar bulge and appear to be spatially offset from the center of a galaxy with indications of recent merging activity. The third row shows the number of AGNs in disturbed, post-merger systems where the stellar bulge is offset from the center of the extended galaxy profile and aligned with the AGN. The fourth row shows the number of AGNs that appear to be point sources spatially offset from an undisturbed galaxy and are therefore more likely to be chance coincidences with background galaxies. The fifth row shows the number of AGNs where the ZTF position could not be confirmed in archival Legacy Survey images.

\subsection{Morphological Classification of AGN Hosts}

In order to confirm the host-AGN offset found in the ZTF images and classify the 251 offset AGNs based on their morphology we undertake Tractor modeling with deeper, higher-resolution images. For this task, we use archival images from the DESI Legacy Imaging Surveys (Dey et al. 2019). The combined DECam Legacy Survey, Mayall $z$-band Legacy Survey, and Beijing-Arizona Sky Survey were taken between 2014 and 2019 and cover declinations of $-18^{\circ}<\delta<+84^{\circ}$, offering 0 ". $262 \mathrm{pix}^{-1}$ resolution and depths of $24.7,23.9$, and 23.0 for the $g, r$, and $z$ bands, respectively.

We model the high-resolution coadded $g_{-}, r$-, and $z$-band Legacy Survey images of each system with a single galaxy profile and an offset point source. We then visually examine the images, Tractor models, and residuals to determine if each offset AGN is well modeled as a point source or if there is excess unmodeled emission indicating the presence of a second host galaxy in the system that is centered on the AGN. We separate the sample into five categories. The number of objects in each category is shown in Table 2. 


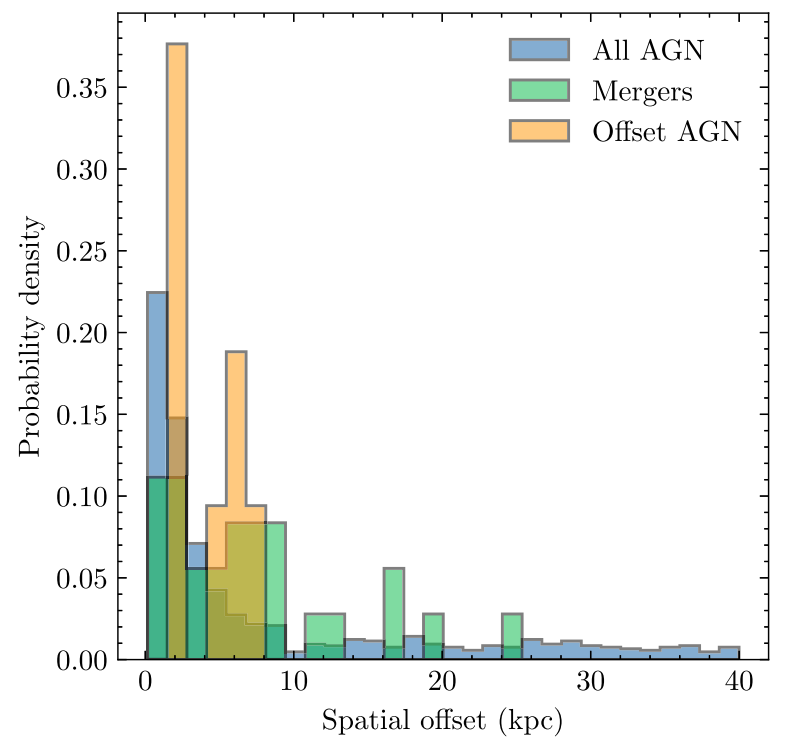

Figure 3. Distribution of physical spatial offsets between AGNs and their closest galaxies for three samples: 898 AGNs from the Tractor-modeled sample with available spectroscopic redshifts, 27 of the 52 AGNs in mergers with spectroscopic redshifts, and the 9 off-nuclear AGNs. A tail of spatial offsets $>10 \mathrm{kpc}$ can be seen in the blue histogram due to the presence of background galaxies.

When the residuals of the galaxy and offset point-source model show a clear stellar bulge surrounding the offset AGN, it is considered likely that there are two galaxies in the system rather than one. If the residuals also show morphological evidence of merging activity such as tidal structures, we consider the AGN to be part of a galaxy merger. These objects will be discussed further in Section 3.1.

When the system is well modeled by a single galaxy profile and offset point source and there are no tidal structures indicating recent merging activity, it is considered likely to be a chance coincidence of an AGN and an unrelated background galaxy. These AGNs are discussed in Section 3.2.

For AGNs that are well modeled by an offset point source and show morphological evidence of recent merging activity in the host galaxy residuals, we consider that the AGN may be a candidate for a recoiling SMBH. We consider these objects as recoiling SMBH candidates because the recoiling AGN is expected to be visible for a period of $10^{6} \mathrm{yr}$ after recoil while the host galaxy will still show evidence of previous merging activity in its morphology (Blecha et al. 2016). These objects are discussed in Section 3.3.

When the AGN is in a disturbed, irregularly shaped host galaxy with a stellar bulge that is offset from the photometric center of the extended galaxy profile and the AGN is aligned with this stellar emission, we do not consider the AGN to be a recoiling SMBH candidate.

The remainder of the 251 objects do not have point-source emission from an AGN present in the Legacy Survey images used for Tractor modeling. For these objects, confirmation of the spatial offset discovered in the ZTF images requires follow-up with deeper, higher-resolution imaging taken when the AGN emission is visible.

The distribution of physical offsets in kiloparsecs for the whole sample, the AGNs in mergers, and the off-nuclear AGNs is shown in Figure 3. The complete AGN sample shows a tail

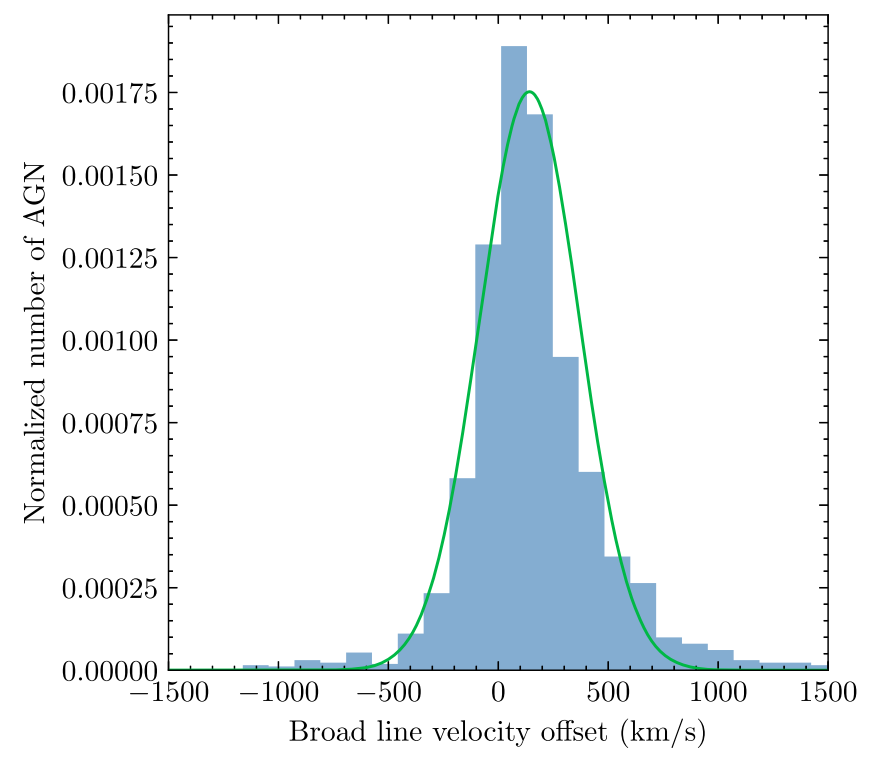

Figure 4. Distribution of Balmer broad-line velocities relative to [S II] $\lambda 6717$, 6731, [N II] $\lambda 6550,6575$, [O I] $\lambda 6302,6366$, and [O III] $\lambda 5007,4959$ emission line velocities found by fitting of archival SDSS spectra with PPXF. Velocities are shown for the 2422 AGNs with broad Balmer lines in archival SDSS spectra out of the complete ZTF AGN sample of 5542 objects.

extending beyond $40 \mathrm{kpc}$ due to chance coincidences with background galaxies.

\subsection{Spectroscopic Analysis of ZTF Broad-line AGNs}

In order to model the distribution of broad-line velocity offsets in the ZTF AGN sample as a whole, we model all 2422 ZTF AGNs that have archival SDSS spectra and are classified as broad-line AGNs by the SDSS DR14 pipeline with penalized pixel fitting (pPXF; Cappellari \& Emsellem 2004; Cappellari 2017). This method finds the velocity dispersion of stellar absorption lines using a large sample of high-resolution templates of single stellar populations adjusted to match the spectral resolution of the input spectrum. We simultaneously fit the narrow $\mathrm{H} \alpha, \mathrm{H} \beta, \mathrm{H} \gamma, \mathrm{H} \delta$, [S II] $\lambda 6717,6731$, [N II] $\lambda 6550$, 6575, [O I] $\lambda 6302,6366$, and [O III] $\lambda 5007,4959$ emission lines during template fitting. The emission line fluxes are each fit as free parameters, but the line widths of the Balmer series are tied, as are the line widths of the forbidden lines.

In these fits the velocity of whichever broad $\mathrm{H} \alpha, \mathrm{H} \beta, \mathrm{H} \gamma$, or $\mathrm{H} \delta$ lines are available within the SDSS spectroscopic wavelength range is allowed to vary up to $3000 \mathrm{~km} \mathrm{~s}^{-1}$ from the narrow lines. The velocity of each Balmer broad line is tied to the other Balmer broad lines.

We find that the ZTF AGN broad-line velocities have a mean displacement of $143 \mathrm{~km} \mathrm{~s}^{-1}$ with a standard deviation of 126 $\mathrm{km} \mathrm{s}^{-1}$ when the central component is fit with a Gaussian (Figure 4). Our distribution of broad-line velocities for variable ZTF AGNs is similar to the distribution of $\mathrm{H} \beta$ broad-line velocities found by Bonning et al. (2007) with a sample of 2598 SDSS AGNs, where they found a mean displacement of $100 \mathrm{~km} \mathrm{~s}^{-1}$ and a standard deviation of $212 \mathrm{~km} \mathrm{~s}^{-1}$.

Our velocity distribution shows a substantial tail population with velocities up to $\pm 2500 \mathrm{~km} \mathrm{~s}^{-1}$. The fractions of AGN $f_{\nu}$ with velocity magnitudes greater than 1000,1500 , and $2000 \mathrm{~km} \mathrm{~s}^{-1}$ are $f_{1000}=0.025, f_{1500}=0.009$, and $f_{2000}=0.003$. These values are comparable to those found in the 
Bonning et al. (2007) sample, where they found fractions of $f_{1000}=0.0035, f_{1500}=0.0012$, and $f_{2000}=0.0008$. It therefore appears that a variability-selected AGN sample shows a broadline velocity distribution that is typical of a spectroscopically selected AGN sample.

\section{Results}

\subsection{AGNs in Galaxy Mergers}

Fifty-two of our spatially offset AGNs are determined to be in a merger with a second galaxy based on the presence of two galaxy nuclei and an interacting morphology visible in Legacy Survey images. The galaxy separations from Tractor modeling of Legacy Survey images range from 0!" 4 to $9^{\prime \prime}$, which for the 33 AGNs with known redshifts correspond to physical separations of $0.54-24.65 \mathrm{kpc}$

For 14 of these AGNs with available SDSS spectra of both galaxies, only ZTF18aacjltc has narrow emission lines consistent with an AGN in both galaxies. The remaining 12 are single AGNs, where the companion galaxy does not show narrow AGN emission line ratios. The observed fraction of dual versus offset AGN is consistent with the predictions of Van Wassenhove et al. (2012).

Eighteen galaxy mergers have only one archival SDSS spectrum available, with the fiber centered on the variable AGN. Follow-up spectra of the companion galaxies will be required to determine if these mergers contain one or two AGNs. Fifteen galaxy mergers have no archival SDSS spectra available, as the variable object was classified as an AGN based on optical ZTF and infrared WISE variability. Follow-up spectra of both the AGN and the companion galaxy will be required to spectroscopically confirm the presence of one or two AGNs.

Aside from appearing in a range of multiwavelength AGN sample papers, a fraction of our sample of AGNs in merging galaxies have been studied in more detail in the literature. ZTF18aasxvyo is a well-studied X-ray-bright BL Lac object (Halpern et al. 1986), and ZTF18aaqjexl is also a known BL Lac object (Plotkin et al. 2008). ZTF18acegbsb is a known double-peaked emitter (Strateva et al. 2003) in a merger, which has been studied in the context of AGN photoionization of companion galaxies (Keel et al. 2019). ZTF18aamfuhc appeared in the same cross-ionization study. ZTF18abhpvvr is a known dual AGN (Huang et al. 2014), and ZTF18aawwfep and ZTF18aajnqqv are also known AGN pairs from Liu et al. (2011).

ZTF18aaifbku, an AGN in the double-lobed galaxy Mrk 783, was imaged with the Karl G. Jansky Very Large Array at $5 \mathrm{GHz}$ on 2015 September 6 and showed radio emission from a compact core component and an extended component that was $26 \mathrm{kpc}$ long (Congiu et al. 2017). The lack of jet emission led the authors to conclude that the radio emission was a relic of previous AGN activity before the AGN entered a quiescent state. This AGN was first observed in ZTF on 2018 June 5 and has shown continued variability to $r$-band magnitudes of 17.07 since then, suggesting that AGN activity has turned on since radio observations were first made in 2015.

In order to determine if ZTF18aaxvmpg is a dual AGN, we undertake more detailed spectroscopic follow-up. The SDSS spectrum of ZTF18aaxvmpg taken on 2006 May 21 with the fiber centered on the AGN shows the presence of broad Balmer lines, but the companion galaxy at $5.5 \mathrm{kpc}$ from the AGN does

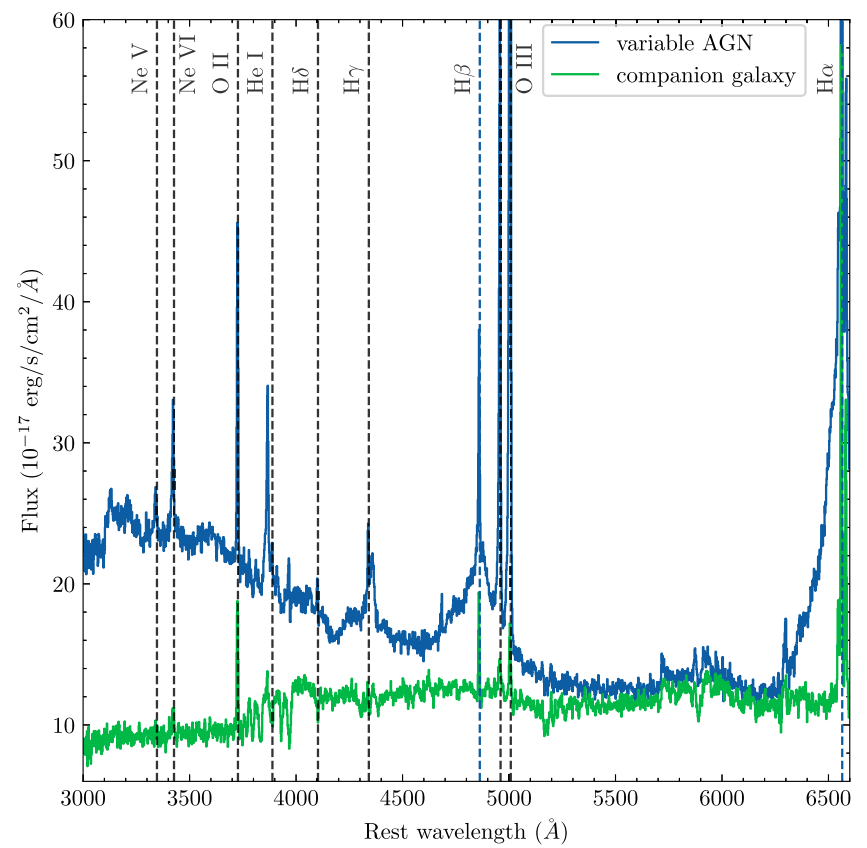

Figure 5. Spectrum of ZTF18aaxvmpg and its offset companion galaxy taken on 2019 October 29 with the DeVeny spectrograph on the LDT. Broad Balmer features can be seen in the AGN spectrum, but only narrow emission lines are visible from the companion galaxy.

not have an archival spectrum. We took a new spectrum of both the host galaxy and the AGN on 2019 October 29 with the DeVeny spectrograph on the LDT using a 1.15 slit, a central wavelength of $5700 \AA$, a spectroscopic coverage of $3600-8000$ $\AA$, and a total exposure time of $3200 \mathrm{~s}$. The spectrum of the AGN and the companion galaxy is shown in Figure 5.

Using the best-fit line fluxes from pPXF modeling of the spectrum, we determine the AGN and host galaxy types using the [O III] $\lambda 5007, \mathrm{H} \beta,[\mathrm{N} \mathrm{II}] \lambda 6573$, and $\mathrm{H} \alpha$ line amplitudes. To separate starburst galaxies, we use the Kauffmann et al. (2003) condition:

$$
\log ([\mathrm{O} \text { III }] / \mathrm{H} \beta)<0.61 /(\log ([\mathrm{N} \text { II }] / \mathrm{H} \alpha)-0.05)+1.3 .
$$

The composite region is defined to be between the above condition and

$$
\log ([\mathrm{O} \text { III }] / \mathrm{H} \beta)<0.61 /(\log ([\mathrm{N} \mathrm{II}] / \mathrm{H} \alpha)-0.47)+1.19 .
$$

The emission line ratios and their classifications for the AGN and companion galaxy are shown in Figure 6. We find that the variable AGN has narrow-line emission consistent with a Seyfert galaxy, while the host galaxy narrow-line emission falls into the composite/LINER category. Since LINER emission can be produced by either AGNs or hot old stars on galactic scales, we must distinguish between the two using a WHAN diagram, which classifies LINERs with a narrow $\mathrm{H} \alpha$ equivalent width $>3 \AA$ and $a \log _{10}([\mathrm{~N}$ II $] \lambda 6573 / \mathrm{H} \alpha)$ flux ratio $>-0.4$ as AGNs (Cid Fernandes et al. 2011; Mezcua \& Domínguez Sánchez 2020). With PPXF we measure the equivalent width of narrow $\mathrm{H} \alpha$ to be $3.54 \pm 0.11 \AA$ and the $\log _{10}([\mathrm{~N}$ II $] \lambda 6573 / \mathrm{H} \alpha)$ flux ratio to be $-0.165 \pm 0.097$. This places the spectrum in the "weak AGN" class of the WHAN diagram, suggesting that the 


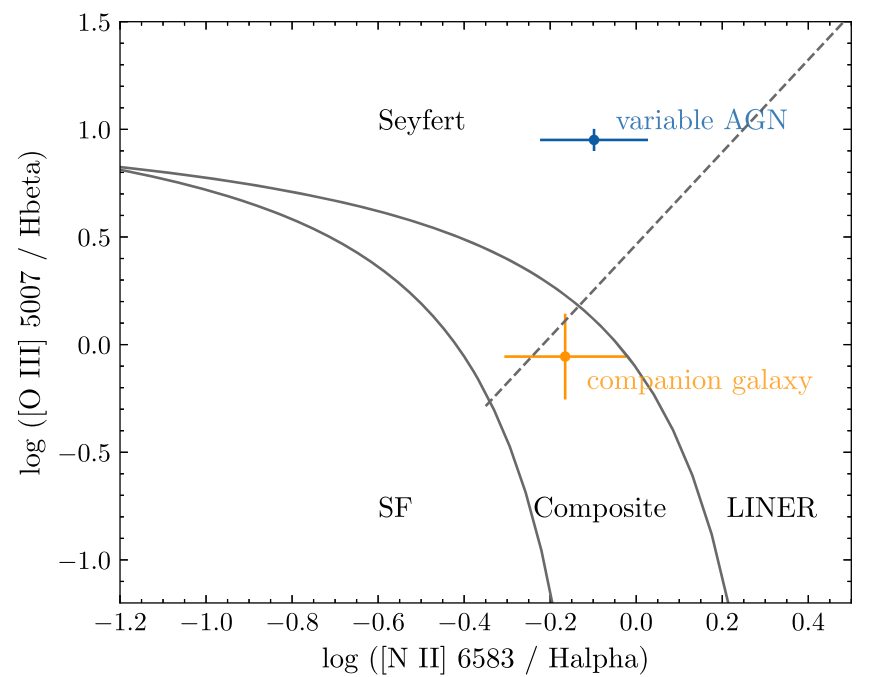

Figure 6. [O III] $\lambda 5007, \mathrm{H} \beta,[\mathrm{N}$ II $] \lambda 6573$, and $\mathrm{H} \alpha$ narrow emission line ratios from pPXF fitting of DeVeny spectra taken of the variable AGN ZTF18aaxvmpg and its companion galaxy overlaid on the AGN classification regions. The ZTF AGN is classified as a Seyfert galaxy, while the offset galaxy has a combination of star-forming and LINER emission.

system does indeed host a second AGN. We therefore conclude that ZTF18aaxvmpg is part of a dual AGN.

Six of the 27 broad-line AGNs in mergers for which we have archival SDSS spectra show double-peaked broad Balmer emission from an unobscured accretion disk, corresponding to $22 \%$ of the sample. The classification of the broad-line region (BLR) shape of each AGN in a merger is shown in Table 3. It will be shown in Section 3.3.1 that $16 \%$ of the broad-line ZTF AGNs are double-peaked emitters. It is therefore possible that AGNs in mergers are more likely to have double-peaked broad lines than normal ZTF AGNs.

In order to determine the X-ray luminosities of the sample, we crossmatch with a $60^{\prime \prime}$ radius to the second ROSAT All-sky Survey catalog (Boller et al. 2016). Thirty-one of the 52 AGNs have X-ray detections. The X-ray fluxes for the 31 AGNs, along with the luminosities for those with a known redshift, are shown in the appendix in Table 6. Nine of the AGNs in mergers have $20 \mathrm{~cm}$ radio detections in Faint Images of the Radio Sky at Twenty cm (FIRST). Six of the 10 AGNs with detected radio emission are also X-ray bright. The smaller fraction of radio AGNs compared to X-ray AGNs may be due to delayed triggering of radio emission during the merger (Shabala et al. 2017; Skipper \& Browne 2018).

The relationship between galaxy separation and X-ray luminosity is shown in Figure 7. We do not find a correlation between galaxy separation and X-ray luminosity in the range of $1-19 \mathrm{kpc}$ separations represented by our sample. While Koss et al. (2012) found that the X-ray luminosity of AGN pairs decreases with increasing separation up to $90 \mathrm{kpc}$, they also did not find a strong correlation at $<20 \mathrm{kpc}$.

In order to compare the variability of the AGNs in mergers with the complete AGN sample, we identify the maximum flux change between the ZTF reference image and the single-epoch science images for the AGNs with spectroscopic redshifts and determine the corresponding change in luminosity. The distribution of peak luminosity change is shown in Figure 8 for the merger sample and for a larger sample of $689 \mathrm{ZTF}$ AGNs, controlled for the quality cuts used to produce the merger sample. The two samples show a similar distribution of peak luminosity. A K-S test to compare the two samples confirms they are drawn from the same distribution with a $p$ value of 0.64 .

\subsection{Chance Coincidences of AGNs and Background Galaxies}

In our sample of offset AGNs we find 29 AGNs that are offset from an undisturbed galaxy with a symmetrically shaped profile. While many of these objects are likely to be quasars coinciding with background galaxies, it is possible that a fraction of them are AGNs with a real association with the spatially offset host. We therefore calculate the approximate number of chance coincidences with background galaxies that we expect for the sample of 3110 AGNs that are modeled by The Tractor.

To determine the density of background galaxies, we use Casjobs to query the SDSS DR16 catalog to find the number of galaxies with $g$-band model magnitudes between 15 and 22.8. We choose a $g$-band limiting magnitude of 22.8 because it is the median effective depth of each set of $30 \mathrm{ZTF}$ images used for Tractor modeling for the AGN sample. We note that the choice of median limiting magnitude is a significant approximation because there is a wide distribution in effective depths for the Tractor models of the AGNs. Future work should take a more systematic approach to reach a consistent limiting magnitude for all objects.

We find that there are 119,364,394 galaxies in the SDSS survey area of $14,555 \mathrm{deg}^{2}$ within this magnitude range, corresponding to a density of $6.328 \times 10^{-4} \operatorname{arcsec}^{-2}$. This means that we expect $1.789 \times 10^{-2}$ background galaxies in a $3^{\prime \prime}$ radius circle around a given AGN. For our sample of 3110 AGNs with Tractor-modeled offsets, we therefore expect 56 AGNs to have unassociated background galaxies within $3^{\prime \prime}$.

As we do not have an excess of AGNs that are intrinsically offset from undisturbed host galaxies beyond the estimated number of chance coincidences, we do not have evidence that any of these objects could be recoiling SMBH candidates. The AGNs falling under this category require spectra to confirm the host galaxy redshift and provide evidence that they are not chance coincidences.

\subsection{AGNs Spatially Offset from the Center of Disturbed Galaxies}

Our morphological classification scheme finds nine AGNs that are variable point sources spatially offset from a potential host galaxy and do not show evidence of a second stellar bulge around them. The properties of the nine offset AGNs are summarized in Table 4. Their ZTF light curves are shown in Figure 9. We show the coadded $g-, r$-, and $z$-band Legacy Survey images, the best-fit Tractor model, and the residuals for each object in Figure 10. The residuals all show asymmetric, tidal structures indicative of previous merging activity. Spectra of the offset AGNs are shown in Figure 11.

\subsubsection{BLR Properties}

The details of the $\mathrm{H} \alpha$ and $\mathrm{H} \beta$ BLRs of the spatially offset AGNs are shown in Figure 12. We do not have $\mathrm{H} \alpha$ and $\mathrm{H} \beta$ spectra for ZTF18absvcae due to its higher redshift. Three of the eight AGNs for which we do have spectra of the $\mathrm{H} \alpha$ or $\mathrm{H} \beta$ lines (ZTF19aadgijf, ZTF18accptjn, and ZTF19aadggaf) have Gaussian broad lines. The broad-line velocity offsets 
Table 3

Galaxy Merger Summary

\begin{tabular}{|c|c|c|c|c|c|c|c|c|}
\hline ZTF Name & $z$ & $\begin{array}{l}\text { R.A. } \\
\text { (hms) }\end{array}$ & $\begin{array}{l}\text { Decl. } \\
(\mathrm{dms})\end{array}$ & $\begin{array}{l}\text { Host-AGN Offset } \\
\text { (arcseconds) }\end{array}$ & $\begin{array}{l}\text { Host-AGN Offset } \\
\qquad(\mathrm{kpc})\end{array}$ & $\begin{array}{l}\text { AGN Flux / Com- } \\
\text { panion Flux }\end{array}$ & $\begin{array}{c}\text { \# AGNs / \# } \\
\text { Spectra }\end{array}$ & $\begin{array}{l}\text { Broad-line } \\
\text { Shape }\end{array}$ \\
\hline ZTF18aaxvmpg & 0.212 & $12: 35: 57.810$ & $58: 21: 21.726$ & $1.296 \pm 0.002$ & $5.477 \pm 0.008$ & 21.25 & $1 / 2$ & $\mathrm{C}$ \\
\hline ZTF18abamzru & $\cdots$ & $17: 23: 27.486$ & $42: 21: 22.683$ & $5.231 \pm 0.001$ & $\ldots$ & 3.20 & $1 / 0$ & $\cdots$ \\
\hline ZTF18aasxvyo & 0.23635 & $14: 17: 56.535$ & $25: 43: 21.506$ & $5.068 \pm 0.001$ & $19.162 \pm 0.004$ & 24.24 & $1 / 2$ & $\cdots$ \\
\hline ZTF18aaieguy & 0.213 & $13: 25: 18.144$ & 41:10:09.717 & $0.676 \pm 0.007$ & $2.88 \pm 0.03$ & 0.48 & $1 / 1$ & $\mathrm{C}$ \\
\hline ZTF19aakjemw & 0.147 & 15:50:07.939 & $27: 28: 10.996$ & $5.435 \pm 0.002$ & $16.184 \pm 0.006$ & 1.16 & $1 / 2$ & $2 \mathrm{~B}$ \\
\hline ZTF18aaifbku & 0.067 & $13: 2: 58.854$ & $16: 24: 27.806$ & $0.985 \pm 0.002$ & $1.371 \pm 0.003$ & 1.75 & $1 / 1$ & $\mathrm{C}$ \\
\hline ZTF18aampabj & 0.216 & $16: 52: 58.864$ & $44: 48: 45.540$ & $3.118 \pm 0.001$ & $13.416 \pm 0.004$ & 15.31 & $1 / 2$ & $\mathrm{C}$ \\
\hline ZTF19aaagygp & $\ldots$ & 01:07:13.787 & $-11: 36: 2.998$ & $3.969 \pm 0.002$ & $\ldots$ & 0.34 & $1 / 0$ & $\cdots$ \\
\hline ZTF18abujubn & $\ldots$ & 19:08:12.617 & $45: 32: 49.643$ & $8.562 \pm 0.002$ & $\ldots$ & 25.97 & $1 / 1$ & MG \\
\hline ZTF18acegbsb & 0.037 & 09:04:36.964 & $55: 36: 02.772$ & $9.027 \pm 0.009$ & $6.992 \pm 0.007$ & 2.14 & $1 / 2$ & RS \\
\hline ZTF19aaozpdm & $\ldots$ & $13: 37: 01.086$ & $20: 25: 14.343$ & $1.682 \pm 0.013$ & $\ldots$ & 0.88 & $1 / 0$ & $\cdots$ \\
\hline ZTF18aacjltc & 0.189 & $08: 12: 52.208$ & $40: 23: 47.253$ & $3.192 \pm 0.001$ & $12.091 \pm 0.004$ & 6.06 & $2 / 2$ & $\mathrm{C}$ \\
\hline ZTF18abtpite & $\ldots$ & $23: 18: 35.915$ & 41:08:0.284 & $3.262 \pm 0.007$ & $\ldots$ & 0.41 & $1 / 0$ & $\cdots$ \\
\hline ZTF18abvwrxu & $\ldots$ & 22:04:07.944 & $-08: 57: 24.505$ & $1.011 \pm 0.003$ & $\cdots$ & 5.87 & $1 / 0$ & $\ldots$ \\
\hline ZTF19abaktpb & $\ldots$ & $16: 42: 19.074$ & $03: 45: 53.037$ & $8.164 \pm 0.008$ & $\ldots$ & 8.5 & $1 / 0$ & $\ldots$ \\
\hline ZTF18aaqjcxl & 0.099 & $07: 58: 47.235$ & 27:05:16.379 & $3.314 \pm 0.001$ & $6.712 \pm 0.002$ & 5.54 & $1 / 1$ & None \\
\hline ZTF18abyoivl & $\ldots$ & $00: 22: 52.018$ & 08:24:0.757 & $0.391 \pm 0.007$ & $\ldots$ & 0.97 & $1 / 0$ & $\ldots$ \\
\hline ZTF18aabdiug & 0.062 & $12: 31: 52.060$ & $45: 04: 43.273$ & $0.572 \pm 0.002$ & $0.738 \pm 0.003$ & 11.13 & $1 / 2$ & $\mathrm{C}$ \\
\hline ZTF19aaviuyv & $\ldots$ & $18: 56: 20.579$ & $37: 12: 36.076$ & $2.651 \pm 0.014$ & $\ldots$ & 3.48 & $1 / 0$ & $\cdots$ \\
\hline ZTF18aabxczq & 0.063 & $10: 38: 33.425$ & 46:58:06.741 & $0.413 \pm 0.003$ & $0.54 \pm 0.004$ & 1.69 & $1 / 2$ & $\mathrm{C}$ \\
\hline ZTF18acvwlrf & 0.233 & $12: 50: 16.219$ & $04: 57: 45.074$ & $1.271 \pm 0.005$ & $5.887 \pm 0.023$ & 0.99 & $1 / 1$ & MG \\
\hline ZTF19aasejqv & 0.233 & $14: 13: 29.817$ & $26: 44: 35.232$ & $1.57 \pm 0.009$ & $7.261 \pm 0.042$ & 2.85 & $1 / 1$ & $2 \mathrm{~B}$ \\
\hline ZTF18aazogyo & 0.081 & $14: 56: 27.421$ & $30: 53: 40.225$ & $5.442 \pm 0.001$ & $9.123 \pm 0.002$ & 13.33 & $1 / 1$ & $2 \mathrm{~B}$ \\
\hline ZTF18aceypvy & 0.163 & 09:51:12.391 & $31: 35: 37.084$ & $2.406 \pm 0.003$ & $7.913 \pm 0.01$ & 9.89 & $1 / 1$ & $\mathrm{C}$ \\
\hline ZTF18acbweyd & 0.189 & $10: 20: 38.565$ & $24: 37: 12.421$ & $4.24 \pm 0.002$ & $16.125 \pm 0.008$ & 11.47 & $1 / 1$ & $\mathrm{C}$ \\
\hline ZTF18acablce & $\ldots$ & $16: 30: 55.490$ & $72: 26: 43.352$ & $1.37 \pm 0.008$ & $\ldots$ & 1.77 & $1 / 0$ & $\ldots$ \\
\hline ZTF18abhpvvr & $\ldots$ & $00: 38: 33.041$ & $41: 28: 53.681$ & $3.595 \pm 0.003$ & $\ldots$ & 9.29 & $1 / 0$ & $\ldots$ \\
\hline ZTF19abfqmjg & $\cdots$ & $22: 56: 41.062$ & $23: 02: 32.510$ & $7.402 \pm 0.003$ & $\cdots$ & 48.85 & $1 / 0$ & $\cdots$ \\
\hline ZTF18abmqwgr & $\ldots$ & $20: 27: 53.382$ & 14:08:50.604 & $1.539 \pm 0.012$ & $\ldots$ & 1.33 & $1 / 0$ & $\ldots$ \\
\hline ZTF19aadgbih & 0.196 & $12: 46: 33.522$ & $45: 34: 21.773$ & $0.381 \pm 0.006$ & $1.494 \pm 0.024$ & 1.82 & $1 / 1$ & $\mathrm{C}$ \\
\hline ZTF19aalpfan & 0.075 & $13: 27: 51.414$ & $06: 42: 49.854$ & $0.627 \pm 0.003$ & $0.964 \pm 0.005$ & 6.86 & $1 / 1$ & $2 \mathrm{~B}$ \\
\hline ZTF18aawwfep & 0.197 & $08: 54: 41.735$ & $30: 57: 54.759$ & $1.37 \pm 0.003$ & $5.424 \pm 0.012$ & 2.22 & $1 / 2$ & $\mathrm{C}$ \\
\hline ZTF19aavxims & $\ldots$ & $12: 48: 55.053$ & $-06: 59: 54.802$ & $4.735 \pm 0.001$ & $\ldots$ & 31.52 & $1 / 0$ & $\cdots$ \\
\hline ZTF19aaaplct & $\ldots$ & $14: 57: 28.940$ & $08: 34: 22.879$ & $2.835 \pm 0.039$ & $\ldots$ & 0.97 & $1 / 0$ & $\ldots$ \\
\hline ZTF18aajnqqv & 0.081 & $12: 57: 41.050$ & $20: 23: 47.747$ & $1.747 \pm 0.004$ & $2.901 \pm 0.007$ & 2.83 & $1 / 2$ & $\mathrm{C}$ \\
\hline ZTF18abszfur & 0.291 & 22:07:16.099 & $12: 11: 03.278$ & $4.322 \pm 0.002$ & $24.646 \pm 0.011$ & 5.16 & $1 / 0$ & $\cdots$ \\
\hline ZTF19abucbkt & 0.16239 & 01:36:04.252 & $21: 37: 25.882$ & $4.60 \pm 0.01$ & $12.935 \pm 0.03$ & 4.47 & $1 / 1$ & $\mathrm{C}$ \\
\hline ZTF18adbhlyb & 0.212 & $11: 17: 59.188$ & $20: 15: 19.078$ & $4.508 \pm 0.001$ & $19.075 \pm 0.004$ & 4.19 & $1 / 1$ & MG \\
\hline ZTF18acxhoij & $\ldots$ & 01:12:07.783 & $-21: 04: 28.682$ & $1.099 \pm 0.045$ & $\ldots$ & 2.66 & $1 / 0$ & $\ldots$ \\
\hline ZTF18acajwep & $\ldots$ & 01:04:05.280 & $21: 22: 31.946$ & $2.906 \pm 0.001$ & $\ldots$ & 7.08 & $1 / 0$ & $\ldots$ \\
\hline ZTF19abipoqj & $\ldots$ & $22: 43: 14.796$ & $80: 59: 27.375$ & $0.499 \pm 0.005$ & $\ldots$ & 0.57 & $1 / 0$ & $\ldots$ \\
\hline ZTF19abpkoou & $\ldots$ & $02: 34: 16.170$ & $05: 18: 42.732$ & $3.524 \pm 0.001$ & $\ldots$ & 8.38 & $1 / 0$ & $\ldots$ \\
\hline ZTF18abztovy & $\ldots$ & $08: 29: 24.624$ & $34: 50: 45.655$ & $1.719 \pm 0.011$ & $\ldots$ & 0.86 & $1 / 0$ & $\ldots$ \\
\hline ZTF18acsllgd & $\ldots$ & $03: 45: 45.495$ & $22: 23: 58.156$ & $1.041 \pm 0.025$ & $\ldots$ & 4.59 & $1 / 0$ & $\ldots$ \\
\hline ZTF19aanxrki & 0.114 & $15: 32: 27.165$ & $04: 19: 22.283$ & $3.557 \pm 0.064$ & $8.304 \pm 0.149$ & 1.29 & $1 / 2$ & $\mathrm{C}$ \\
\hline ZTF18aamfuhc & 0.086 & $13: 42: 34.214$ & $19: 13: 34.184$ & $4.845 \pm 0.001$ & $8.608 \pm 0.002$ & 28.39 & $1 / 1$ & MG \\
\hline ZTF18aadwvyr & 0.126 & $08: 29: 44.346$ & $32: 52: 21.163$ & $0.984 \pm 0.003$ & $2.53 \pm 0.008$ & 2.15 & $1 / 1$ & $\mathrm{C}$ \\
\hline ZTF19abauzsd & 0.285 & $15: 54: 32.681$ & $21: 43: 48.220$ & $0.483 \pm 0.007$ & $2.699 \pm 0.039$ & 1.03 & $1 / 1$ & RS \\
\hline ZTF18abufbsq & $\ldots$ & $23: 46: 15.513$ & $12: 47: 07.733$ & $0.891 \pm 0.003$ & $\ldots$ & 1.14 & $1 / 0$ & $\ldots$ \\
\hline ZTF18abzuzrg & 0.178 & $16: 55: 16.540$ & $32: 15: 55.145$ & $1.369 \pm 0.007$ & $4.908 \pm 0.025$ & 1.11 & $1 / 2$ & MG \\
\hline ZTF18abtmcdb & $\ldots$ & $01: 20: 12.473$ & $07: 12: 58.251$ & $0.535 \pm 0.003$ & $\ldots$ & 3.81 & $1 / 0$ & $\ldots$ \\
\hline ZTF18aauhnby & 0.09 & $12: 04: 15.954$ & $56: 02: 58.100$ & $1.092 \pm 0.002$ & $2.02 \pm 0.004$ & 1.39 & $1 / 1$ & $\mathrm{C}$ \\
\hline
\end{tabular}

Notes. Summary of the properties of the 52 AGNs in galaxy mergers. The host-AGN spatial offset and $g$-band AGN/host galaxy flux ratio are derived from Tractor modeling of Legacy Survey images. The eighth column lists the number of AGNs in the system, confirmed by either spectroscopic narrow emission line ratios or the presence of WISE variability. The second number in this column indicates whether neither the AGN nor the host galaxy has a spectrum (0), only the AGN has a spectrum (1), or there is a spectrum of the host galaxy centroid available as well (2). The last column shows the classification of the shape of the BLR, where we adopt the scheme of Strateva et al. (2003): prominent red shoulder (RS), prominent blue shoulder (BS), two prominent peaks (2P), two blended peaks (2B), and complex multi-Gaussian structure (MS). We denote a Gaussian broad line with "C" and an AGN with no broad-line emission with "None." If there are no SDSS spectra available for the source or the source does not have broad emission lines, we indicate this with "...". 


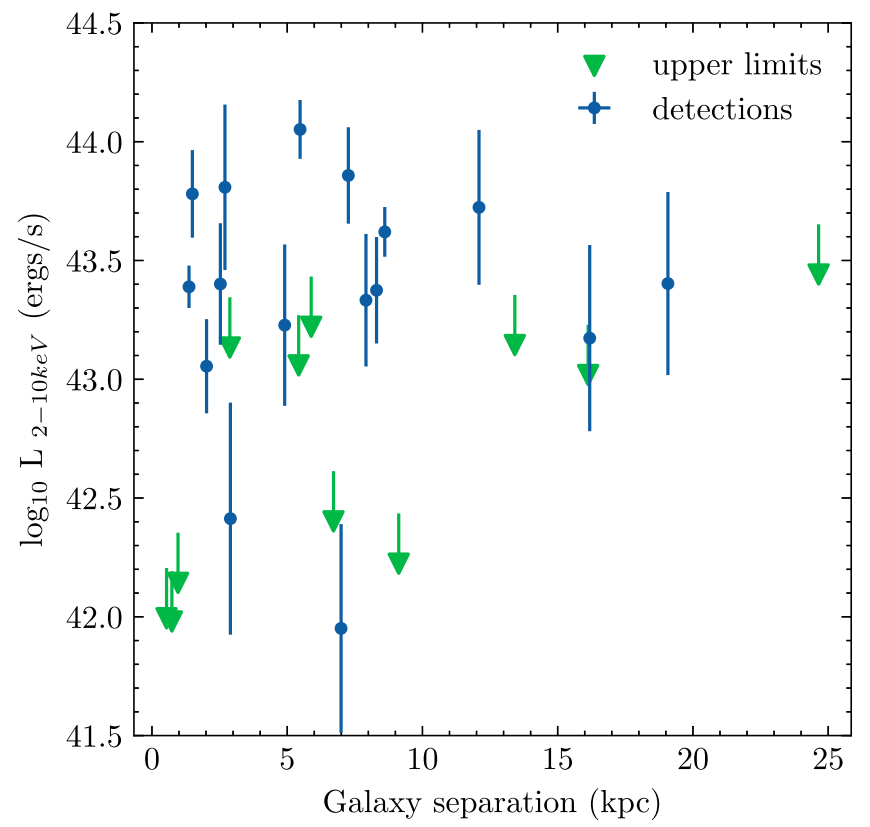

Figure 7. $2-10 \mathrm{keV}$ luminosity for 27 AGNs with known spectroscopic redshifts as a function of physical galaxy separation. Sixteen are detected in the second ROSAT All-sky Survey catalog, and 11 have nondetections.

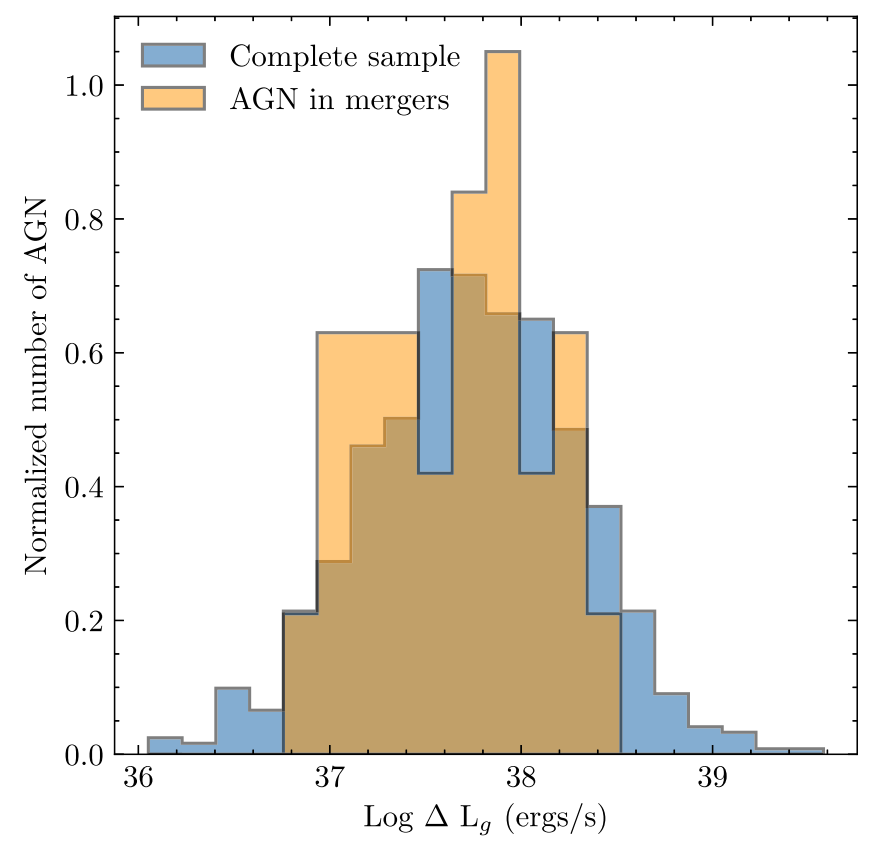

Figure 8. Peak luminosity change relative to the reference image among the 27 AGNs in mergers and a larger sample of 689 variable AGNs with available spectroscopic redshifts. The larger sample is controlled to undergo the same quality cuts as the merger sample.

determined from PPXF fitting are shown in Table 4. Because of the large distribution of velocity offsets in the overall ZTF AGN sample (Figure 4) and the fact that the ZTF AGNs that have extreme $>1500 \mathrm{~km} \mathrm{~s}^{-1}$ velocities do not show any evidence of a spatial offset, we do not make any conclusions from the velocities of $200-600 \mathrm{~km} \mathrm{~s}^{-1}$ magnitude observed for these three AGNs.

The remaining five of the eight AGNs (ZTF18aalsidi, ZTF19aautrth, ZTF18aaxmrom, ZTF19aayrjsx, and ZTF18aaoeobb) for which we have spectra of the $\mathrm{H} \alpha$ or $\mathrm{H} \beta$ lines have asymmetric Balmer BLRs that are poorly fit by a Gaussian. Adopting the classification scheme of Strateva et al. (2003) we note that ZTF18aalsidi and ZTF18aaxmrom have prominent red shoulders; ZTF18aautrth has a prominent blue shoulder; ZTF19aayrjsx has two prominent peaks, which are red- and blueshifted; and ZTF18aaoeobb has two blended peaks, which are red- and blueshifted. These classifications are displayed in Table 4. Such asymmetric structures can arise due to double-peaked emission from an unobscured, relativistic Keplerian accretion disk (Chen et al. 1989; Chen \& Halpern 1989; Eracleous \& Halpern 1994).

To determine if the velocity offset peaks observed in the broad Balmer lines of ZTF19aautrth, ZTF18aaxmrom, ZTF19aayrjsx, ZTF18aaoeobb, and ZTF18aalsidi can be accounted for by accretion disk emission, we model the broad lines with an elliptical accretion disk model where an inner thick hot-ion torus illuminates a thin outer disk of ionized gas, which has a power-law relation between emissivity and radius given by slope $q$ (Chen et al. 1989; Strateva et al. 2003). The model depends on the inner and outer dimensionless gravitational radii of the disk $\epsilon_{1}$ and $\epsilon_{2}$; the local turbulent broadening parameter $\sigma$; the azimuthal angle $\phi$; the inclination angle $i$, where $0^{\circ}$ is face-on and $90^{\circ}$ is edge-on; the ellipticity $e$; and the disk orientation $\phi_{0}$. We bound the inner radius to $0<\epsilon_{1}<1000$, the outer radius to $0<\epsilon_{2}<8000$, the emissivity slope to $0<q<5$, and the local broadening to $0<\sigma<0.01$ to ensure that we could find good fits to the data with disk shape parameters consistent with the known double-peaked emitter population from Strateva et al. (2003). All four objects require a central Gaussian broad line in addition to the accretion disk model to produce a good fit to the data, which is not uncommon for double-peaked emitters (Strateva et al. 2003).

The best-fit disk parameters from the $\mathrm{H} \alpha$ and $\mathrm{H} \beta$ fits of the five sources are shown in Table 5, and the models are shown in Figure 13. We note that our simple log-likelihood minimization procedure for disk model fitting may not explore the whole parameter space and find the most optimal disk parameters. However, it serves to illustrate that accretion disk emission models can account for the extra flux that is not well described by a Gaussian model. More rigorous fitting with Markov Chain Monte Carlo could be used to better determine disk shape parameters and their uncertainties in the future.

In order to determine if the double-peaked emitter fraction of $63 \%$ among spatially offset AGNs is substantially different from the entire variable ZTF AGN sample, we apply a multiGaussian fitting procedure to the 1923 variable AGNs with archival SDSS spectra of the $\mathrm{H} \alpha$ region that have broad Balmer lines to search for double-peaked emitters. We use the pPXF fitting procedure described in Section 2.4 to fit two different models. In the first model the spectrum is fit with a single broad Balmer line free to have a velocity offset to the narrow Balmer lines. This is fit at the same time as the narrow emission lines and stellar continuum. In the second model the spectrum is fit with three broad lines: one with the central velocity tied to the narrow Balmer lines, one with a velocity up to $6000 \mathrm{~km} \mathrm{~s}^{-1}$ relative to the narrow lines, and one with a velocity down to $-6000 \mathrm{~km} \mathrm{~s}^{-1}$. The widths of the three broad lines are not tied to each other.

To find double-peaked emitters from the fits of these two models, we require the $\chi^{2}$ improvement from the multiplebroad-line model compared to the single-broad-line model to 
Table 4

Properties of the Offset AGN Sample

\begin{tabular}{|c|c|c|c|c|c|c|c|c|c|}
\hline ZTF Name & $z$ & $\begin{array}{l}\text { R.A. } \\
\text { (hms) }\end{array}$ & $\begin{array}{l}\text { Decl. } \\
\text { (dms) }\end{array}$ & $\begin{array}{l}\text { Host-AGN Offset } \\
\text { (arcseconds) }\end{array}$ & $\begin{array}{l}\text { Host-AGN Offset } \\
(\mathrm{kpc})\end{array}$ & $\begin{array}{l}\text { BLR Velocity } \\
\left(\mathrm{km} \mathrm{s}^{-1}\right)\end{array}$ & X-Ray? & $\begin{array}{l}\text { Radio Flux } \\
(\mathrm{mJy} / \text { beam })\end{array}$ & $R$ \\
\hline ZTF19aautrth & 0.208 & 16:30:41.964 & $30: 36: 2.448$ & $1.076 \pm 0.003$ & $4.48 \pm 0.012$ & $\mathrm{BS}+\mathrm{C}$ & $\checkmark$ & $<0.98$ & $<1.56$ \\
\hline ZTF18aaxmrom & 0.347 & 16:09:11.257 & $17: 56: 16.271$ & $1.057 \pm 0.01$ & $7.089 \pm 0.067$ & $\mathrm{RS}+\mathrm{C}$ & $\checkmark$ & 444.48 & 1760 \\
\hline ZTF19aayrjsx & 0.215 & 23:32:54.463 & 15:13:5.407 & $1.329 \pm 0.003$ & $5.695 \pm 0.013$ & $2 \mathrm{P}+\mathrm{C}$ & $\checkmark$ & $\cdots$ & $\ldots$ \\
\hline ZTF18aalsidi & 0.348 & 15:53:57.736 & $47: 52: 32.015$ & $0.901 \pm 0.004$ & $6.051 \pm 0.027$ & $\mathrm{RS}+\mathrm{C}$ & $\checkmark$ & $<0.88$ & $<10.32$ \\
\hline ZTF18accptjn & 0.214 & 22:12:17.117 & 3:50:40.531 & $0.648 \pm 0.005$ & $2.767 \pm 0.021$ & $344.3 \pm 0.3$ & $x$ & 1.12 & 0.87 \\
\hline ZTF19aadggaf & 0.266 & $13: 42: 06.57$ & $5: 05: 23.898$ & $0.488 \pm 0.004$ & $2.554 \pm 0.021$ & $608.2 \pm 0.3$ & $\checkmark$ & 3.80 & 2.89 \\
\hline
\end{tabular}

Notes. Spectroscopic and multiwavelength properties of the sample of AGNs spatially offset from disturbed host galaxies. The redshifts are based on the position of O III lines in the archival SDSS spectrum of the AGN. The spatial offsets are obtained from modeling of point-source and galaxy profile positions in the Legacy Survey images with Tractor. For the three AGNs with Gaussian broad lines the broad-line velocities are found by modeling a Gaussian Balmer broad-line series with PPXF. For objects that are poorly modeled by a Gaussian and well modeled by a double-peaked accretion disk model (Chen et al. 1989) we instead classify the shape of the broad line by adopting the scheme of Strateva et al. (2003): prominent red shoulder (RS), prominent blue shoulder (BS), two prominent peaks (2P), and two blended peaks (2B). If a central Gaussian broad line is required in addition to the double-peaked accretion disk model, we indicate this with "+C." The $\mathrm{X}$-ray detection column shows which objects have detections within $1 ! 0$ of the AGN position in the ROSAT All-sky Survey catalog, and the radio detection column shows the $20 \mathrm{~cm}$ flux density or upper limit if it was within the coverage of the FIRST radio survey (Helfand et al. 2015). The radio-loudness is indicated in the last column.

be $>250$. Then to be considered a double-peaked emitter candidate the two velocity offset $\mathrm{H} \alpha$ broad lines are each required to have a peak flux density of $>33 \%$ of that of the narrow $\mathrm{H} \alpha$ line and to have a velocity of $>500 \mathrm{~km} \mathrm{~s}^{-1}$ from the narrow-line velocity. As the effectiveness of these criteria depends on the relative brightness of the narrow and broad lines and on the width of the BLR, we visually inspect the 275 candidates found via these criteria and reject 82, leaving 193 double-peaked emitters. We then visually inspect the remaining 1648 spectra to find any objects that may have been missed by the criteria. We find 106 double-peaked spectra that were missed by the automatic classification scheme.

We therefore estimate that 299 of the 1923 variable AGNs $(16 \%)$ are double-peaked emitters. This is much larger than the 3.6\% fraction found for SDSS AGNs by Strateva et al. (2003) with spectroscopic principal component analysis, suggesting that variable AGNs are more likely to be double-peaked emitters or that our classification scheme is more likely to classify asymmetric BLRs as double-peaked emitters than classification schemes used in previous studies. The $16 \%$ fraction of variable ZTF AGNs with double-peaked broad Balmer lines is substantially smaller than the $63 \%$ fraction seen in the spatially offset AGN sample.

\subsubsection{Multiwavelength Properties}

Seven of the nine offset AGNs are detected in the ROSAT All-sky Survey, and four AGNs (ZTF18aaxmrom, ZTF18accptjn, ZTF18absvcae, and ZTF19aadggaf) are detected at $20 \mathrm{~cm}$ wavelengths in the FIRST radio survey, which uses the Very Large Array to image a footprint largely coincident with SDSS to a detection sensitivity of $1 \mathrm{mJy}$ (Helfand et al. 2015). The radio fluxes for these four AGNs, as well as the upper limits for another four that are within FIRST survey coverage but are not detected, are shown in Table 4. The contours of the FIRST radio imaging are overlaid in Figure 10. ZTF18aaxmrom shows two radio lobes on either side of the galaxy, which are likely the result of synchrotron emission from a jet. The other three AGNs show radio point sources that coincide with the position of the ZTF AGN. The radio-loudness ${ }^{15}$ is also shown in Table 4. Two AGNs with radio emission are classified as radio-loud $(R>10$, Kellermann et al. 1989), two are radio-moderate, and four others have upper limits indicating that they are not radio-loud.

The recoiling SMBH candidates from Chiaberge et al. (2017) and Lena et al. (2014) also demonstrate radio emission. As noted by Chiaberge et al. (2017), radio emission from a recoiling $\mathrm{SMBH}$ is not surprising given that the rapid spin needed to produce a relativistic jet can be produced by a binary black hole merger (Hemberger et al. 2013; Schnittman 2013) and that there is a link between radio-loud AGNs and major galaxy mergers (Ivison et al. 2012; Chiaberge et al. 2015). Assuming that the radio jet axis matches the spin axis of the recoiling $\mathrm{SMBH}$, we would also expect the recoil velocity to be preferentially aligned with the radio jet (Lena et al. 2014).

\subsubsection{Confirming the Nature of the Offset AGNs}

There are two main alternative explanations for the nature of these offset AGNs. First, they may be chance coincidences with disturbed background galaxies. Second, they may be AGNs in mergers with compact or undermassive host galaxies such that extended emission around the AGN is very faint. Given the predicted frequency of SMBHs in tidally stripped nuclei, which may appear as offset AGNs (Voggel et al. 2019), it would not be surprising if a large fraction of our objects are in fact AGNs in compact galaxies merging with a larger galaxy.

If the distribution of expected velocity and spatial offsets of the recoiling SMBH population follows the simulated distribution of Blecha et al. (2016), we would expect the velocity offsets of our sample to range from 300 to $3000 \mathrm{~km} \mathrm{~s}^{-1}$ given their large spatial offsets. Spectroscopic fitting (Section 3.3.1) shows that the three AGNs with Gaussian broad lines have velocity offsets ranging from 344 to $608 \mathrm{~km} \mathrm{~s}^{-1}$ (see Table 4) and the remainder of the sample with double-peaked emission have spectra consistent with a BLR and accretion disk close to

$\overline{15} R=\frac{L_{5 \mathrm{GHz}}(\nu)}{L_{4400 \AA}(\nu)}$. 

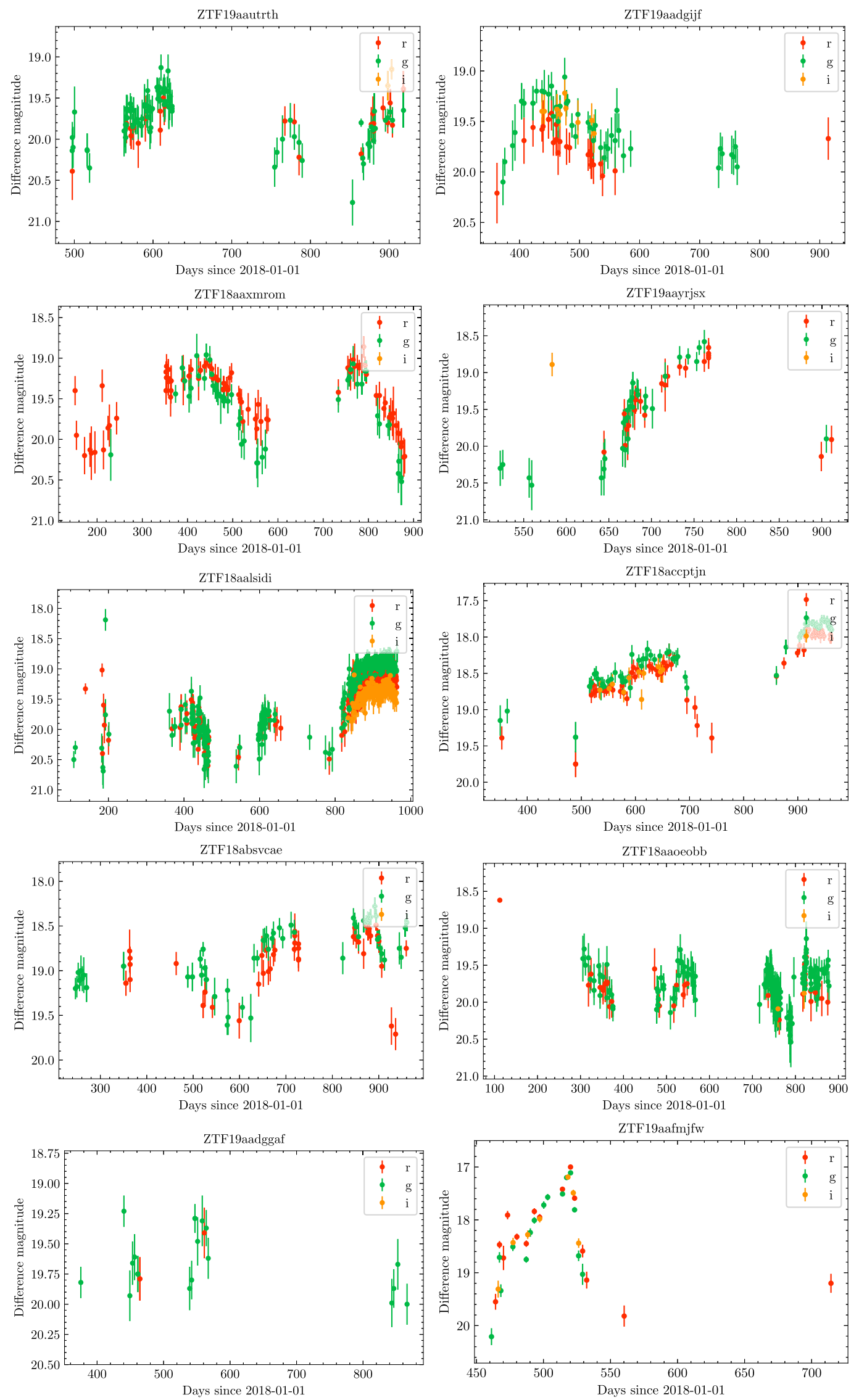

Figure 9. ZTF light curves in $g, r$, and $i$ bands for the sample of nine offset AGNs and the known recoiling SMBH candidate in SDSS 1133 that rebrightens in ZTF (ZTF19aafmjfw). We show only the $>3 \sigma$ detections. 

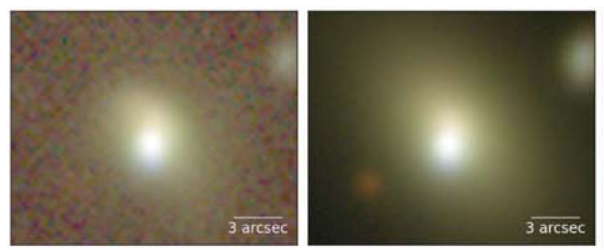

(a) ZTF19aautrth
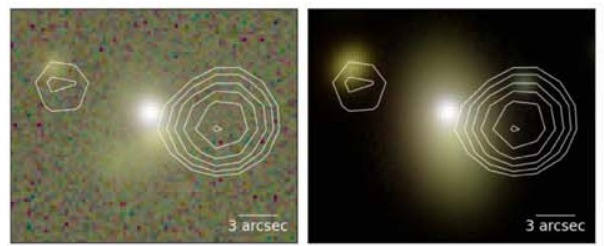

(c) ZTF18aaxmrom
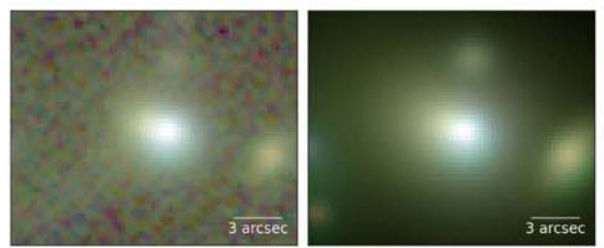

(e) ZTF18aalsidi
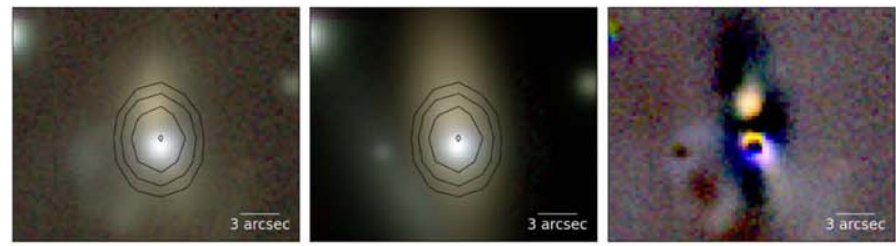

(g) ZTF18absvcae
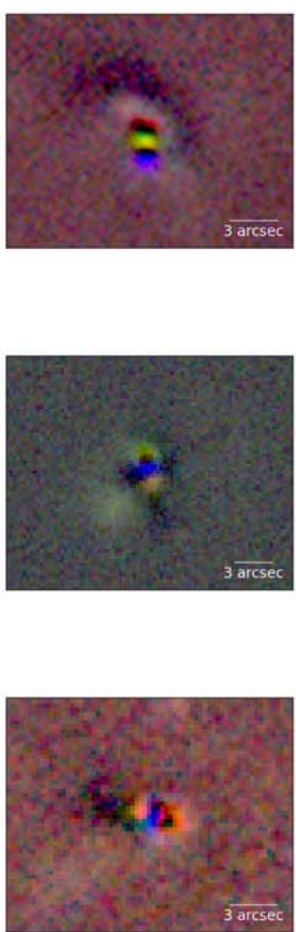
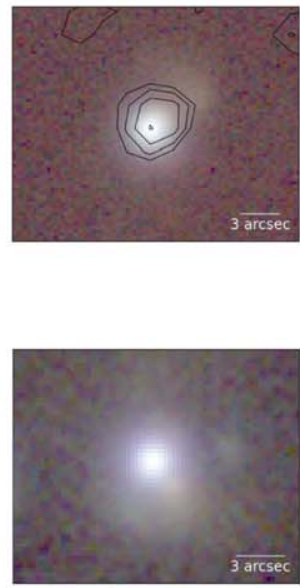

(h) ZTF18aaoeobb

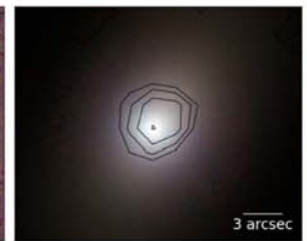

(f) ZTF18accptjn
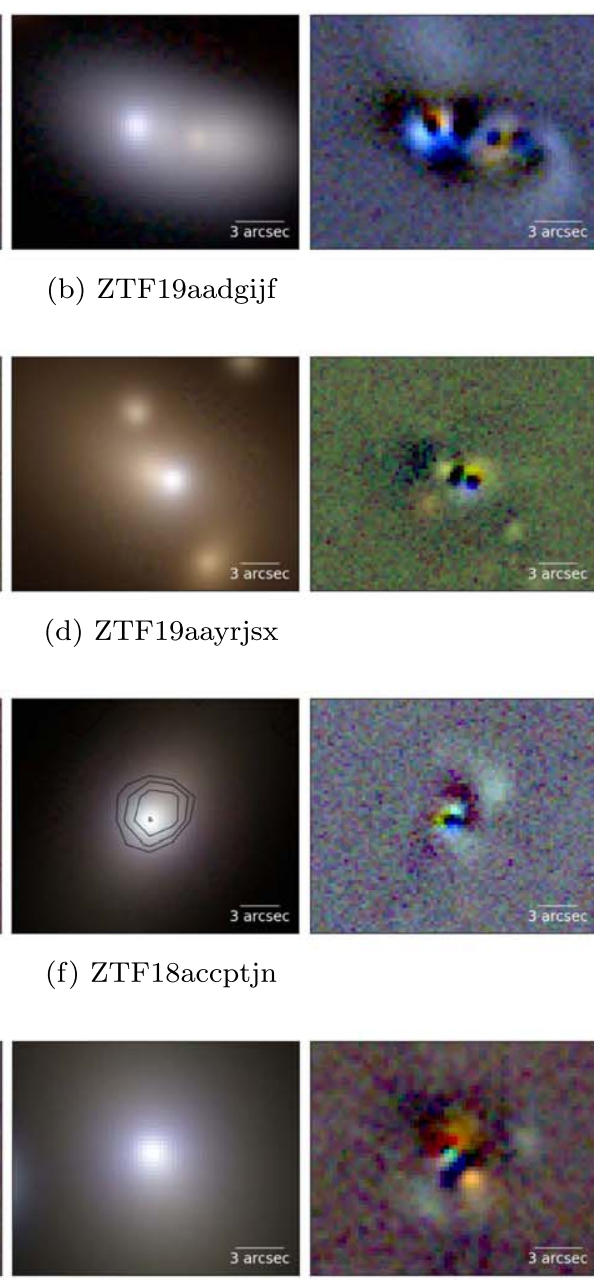

(b) ZTF19aadgijf
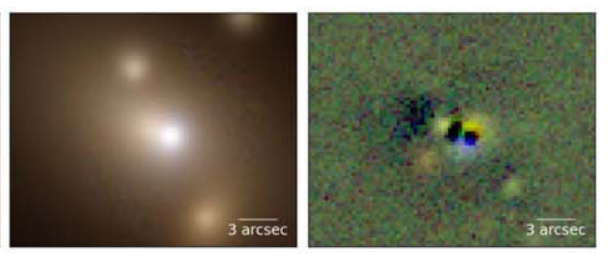

(d) ZTF19aayrjsx
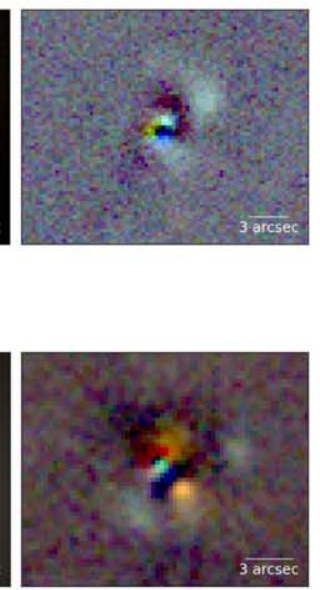

$\widehat{\operatorname{arcsec}}$
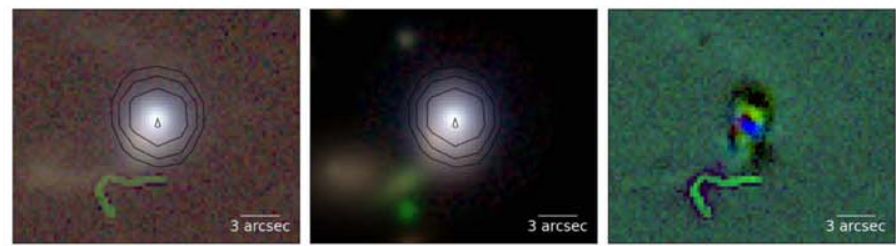

(i) ZTF19aadggaf

Figure 10. For each offset AGN: Left: Coadded $g-, r$-, and $z$-band Legacy Survey images. Middle: Corresponding coadded Tractor model. Right: Image-model residuals showing galaxy tidal structures. For the four AGNs with $20 \mathrm{~cm}$ detections in FIRST (ZTF18aaxmrom, ZTF18accptjn, ZTF18absvcae, and ZTF19aadggaf) we overlay the contours of the FIRST image.

rest velocity (Figure 13). It is possible for large-separation recoiling SMBHs to show low line-of-sight velocities, depending on their orbital trajectory at the time. However, if our objects are indeed all recoiling SMBHs, it is surprising that a fraction of the nine do not have higher $>600 \mathrm{~km} \mathrm{~s}^{-1}$ line-ofsight velocities. The observed velocities therefore argue against the recoil hypothesis for these objects.

Much further work is therefore required to investigate the recoiling SMBH hypothesis for any of these offset AGNs. In order to rule out that they are not AGNs in mergers with undermassive host galaxies, HST infrared imaging could be used to search for a second extended region of old stellar emission around the offset AGN. HST imaging would be well complemented by integral field unit observations to map the positions of the narrow-line emission relative to the AGN broad-line emission (Chiaberge et al. 2018).

Chandra X-ray imaging to search for a second obscured AGN would also be essential for these candidates (Comerford et al. 2017). If we observe just one $\mathrm{X}$-ray point source, we can produce upper limits on the X-ray luminosity of a second AGN and show that they rule out the presence of an obscured AGN using the [O III] flux from the host center. This would strongly favor the recoiling black hole hypothesis.

As spatial offsets greater than $1.5 \mathrm{kpc}$ are only possible in simulations where misaligned SMBH binary spins can occur (Blecha et al. 2016), if even just a subset of our candidates 


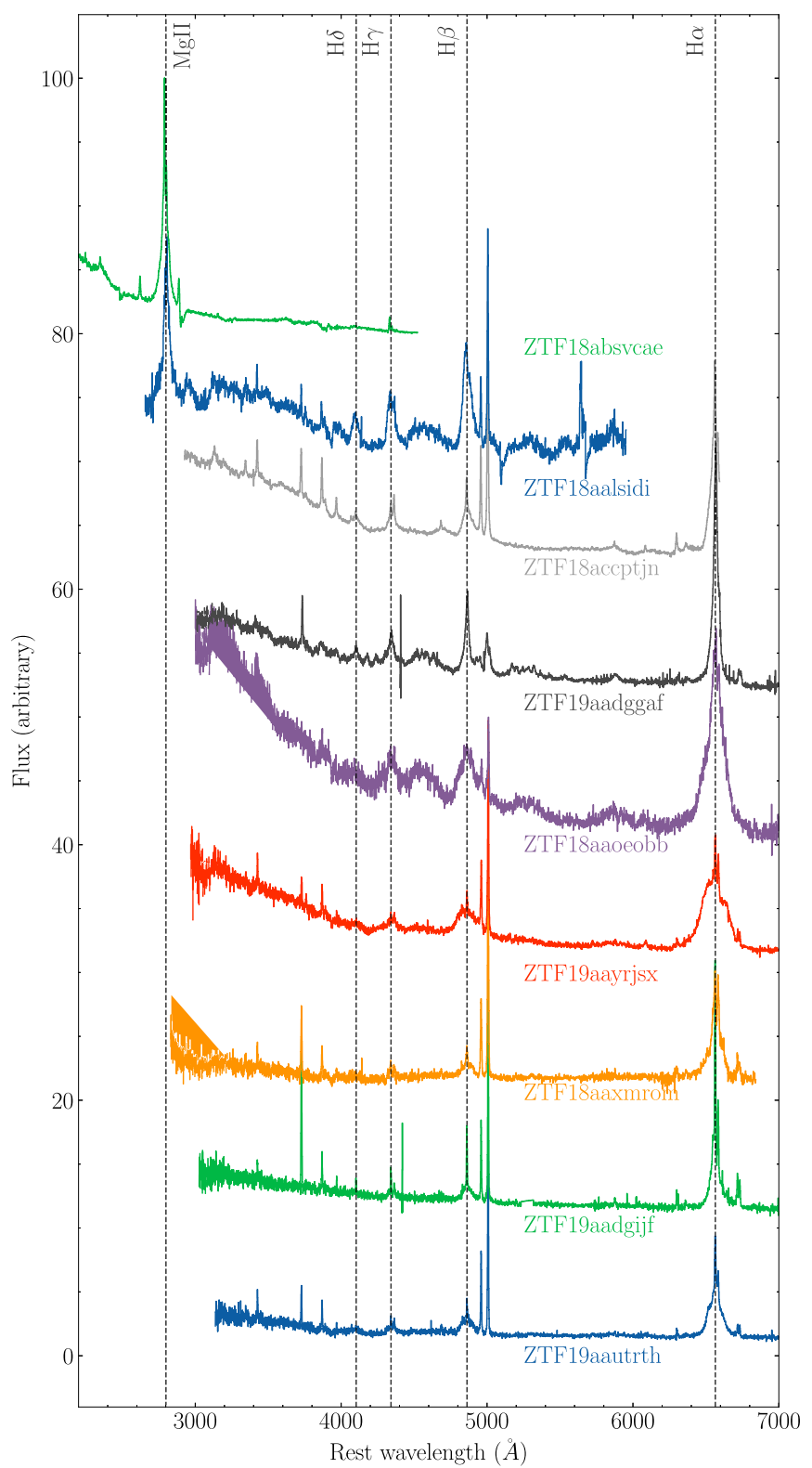

Figure 11. Spectra of the off-nuclear AGNs, all from the SDSS archive except for ZTF18absvcae, ZTF18accptjn, and ZTF18aalsidi, which were observed with the DeVeny spectrograph on the LDT on 2020 September 13 and 15 and 2020 October 11, respectively. In all the SDSS spectra, the fiber was centered on the AGN rather than on the photometric center of the offset host galaxy. The Balmer series is shown with dotted lines.

could be confirmed as recoiling SMBHs, their extreme spatial offsets could demonstrate the existence of SMBH binaries with misaligned spins.

\subsection{SDSS 1133 (ZTF19aafmjfw)}

SDSS 1133, a variable object and recoiling SMBH candidate discovered by Koss et al. (2014), is identified by our search pipeline during a dramatic flare in ZTF, labeled ZTF19aafmjfw. This object has a long history of variability, and the alternative scenarios proposed for its origin include an LBV that exploded as a Type IIn supernova in 2001, an LBV that continues to exhibit giant eruptions, and an offset AGN with flaring and stochastic variability (Koss et al. 2014). It was first observed in 1950 with the $103 \mathrm{aO}$ Digitized Sky Survey plate at a magnitude of 18.6 and was again observed at comparable magnitudes in 1994 and 1999. It was then discovered to be flaring to $g=16.4$ in SDSS on 2001 December 18 and 2002 April 1 but had faded to $g=18.7$ by 2003 March 9. It was observed at a minimum of $g=20.18$ with PS1 on 2012 February 22, after which it brightened to $g=19.3$ on 2014 January 20 (Koss et al. 2014).

We search for this object in archival data from the Catalina Real-time Transient Survey (Drake et al. 2009) and find that this object demonstrated another small-scale flaring event in 2014. After showing no detectable $V$-band activity between 2006 February 3 and 2014 May 12, it brightened by $V=0.22$ between 2014 April 28 and 2014 June 5. After a gap in observations it had faded again by 2014 December 27.

This object showed no evidence of variability in ZTF prior to 2019 April 7, when it became detectable at $m_{g}=20.21$; it flared to $m_{g}=17.00$ on 2019 June 5 and faded again by 2019 December 16. The ZTF flare ZTF19aafmjfw is shown in Figure 9. The $3 \mathrm{mag}$ change in brightness seen in ZTF is of comparable scale to the SDSS flare in 2001. The ZTF rebrightening of this object suggests that the transient emission from 2001 to 2013 was not from a Type IIn supernova with a variable-star progenitor.

We obtained a spectrum of ZTF19aafmjfw with the DeVeny spectrograph on the LDT on 2019 May 29 when the object was bright. The spectrum showed the return of broad $\mathrm{H} \alpha$ and $\mathrm{H} \beta$ absorption lines blueshifted over a $2000-8000 \mathrm{~km} \mathrm{~s}^{-1}$ range (Figure 14), as had been observed in 2013 December to 2014 January (Koss et al. 2014). It also showed the return of [Fe II] $\lambda 7155$ and [Ca II] $\lambda \lambda$ 7291, 7324 lines-features that are seen only occasionally in AGN spectra (e.g., Phillips 1976), latetime supernova spectra (e.g., Filippenko 1997; Pastorello et al. 2019), and outbursting LBVs (Solovyeva et al. 2019).

The 100 day timescale, 3-4 mag flux change, and highvelocity absorption lines make this outburst comparable to the transient SN 2009ip, a supersonic stellar explosion from a $>60 M_{\odot}$ star (Smith et al. 2010; Foley et al. 2011). The presence of broad Balmer, He I, and Na D lines and a very blue continuum also links ZTF19aafmjfw to SN 2009ip. However, SN 2009ip does not show the forest of Fe-group lines and [Ca II] $\lambda \lambda 7291,7324$ emission lines that we observe in ZTF19aafmjfw. Such $\mathrm{Fe}$ and $\mathrm{Ca}$ features were observed in the stellar outburst UGC 2773 OT2009-1, which is likely to be either a giant LBV eruption or extreme S Dor variability with circumstellar dust eruption (Smith et al. 2010; Foley et al. 2011). However, UGC 2773 OT2009-1 has a cooler temperature and weaker Balmer emission than SN 2009ip, suggesting that it was a subsonic outburst rather than an explosion.

As ZTF19aafmjfw has spectroscopic features in common with SN 2009ip, which could indicate a high-velocity explosion (blueshifted Balmer absorption lines, bright and broad Balmer emission, and a blue continuum), and other spectroscopic features in common with UGC 2773 OT2009-1, which could indicate a stellar outburst within a circumstellar dust envelope (Fe II and Ca II emission lines), the observed spectroscopic features may instead be due to a nonterminal, supersonic LBV outburst in a dusty circumstellar environment.

We note, however, that the presence of such a rare and massive star in a dwarf galaxy that does not show recent star formation in Keck adaptive optics and HST imaging is very unlikely (Koss et al. 2014), and while the observed combination of spectroscopic features would be unusual for a standard 

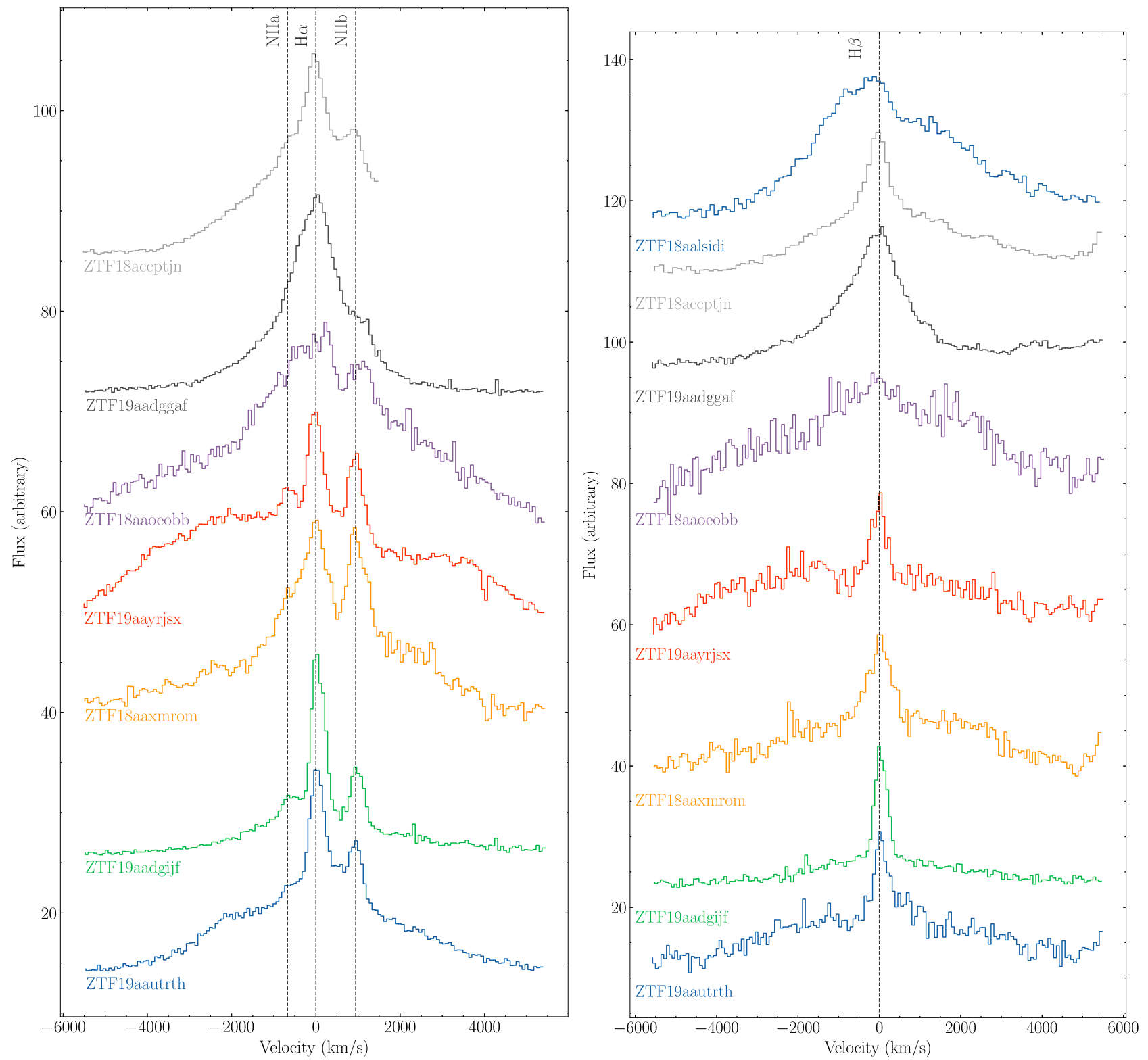

Figure 12. $\mathrm{H} \alpha$ BLRs (left) and $\mathrm{H} \beta$ BLRs (right) for the off-nuclear AGN candidates. Five of the eight off-nuclear AGNs for which we have spectra of either the H $\alpha$ or the $\mathrm{H} \beta$ lines show asymmetric or double-peaked broad Balmer structures (ZTF18aaoeobb, ZTF19aayjrsx, ZTF18aaxmrom, ZTF19aautrth, and ZTF18aalsidi). The other three show standard Balmer broad lines (ZTF18accptjn, ZTF19aadggaf, and ZTF19aadgijf).

Table 5

Best-fit Elliptical Accretion Disk Parameters

\begin{tabular}{|c|c|c|c|c|c|}
\hline Disk parameter & ZTF19aautrth & ZTF18aaxmrom & ZTF19aayrjsx & ZTF18aaoeobb & ZTF18aalsidi \\
\hline Central component required & Yes & Yes & Yes & Yes & Yes \\
\hline Inner radius $(\epsilon)$ & 252 & 813 & 70 & 997 & 21 \\
\hline Outer radius $(\epsilon)$ & 608 & 6800 & 7995 & 3841 & 2255 \\
\hline Inclination (degrees) & 13.6 & 84.9 & 14.0 & 47.6 & 12.8 \\
\hline Turbulent broadening $(c)$ & 0.0035 & 0.0007 & 0.007 & 0.0019 & 0.0042 \\
\hline Ellipticity & 0.46 & 0.54 & 0.91 & 0.17 & 0.93 \\
\hline Disk orientation (deg) & 18.2 & 36.6 & 39.0 & 31.6 & 357.1 \\
\hline
\end{tabular}

Notes. Best-fit accretion disk parameters for the five double-peaked emitters from modeling of the H $\alpha$ (ZTF19aadgijf, ZTF19aautrth, ZTF18aaxmrom, and ZTF19aayrjsx) and $\mathrm{H} \beta$ (ZTF18aalsidi) double-peaked emission after subtracting a stellar continuum model derived from pPXF. The disk parameters are derived from a simple log-likelihood minimization fit to illustrate that accretion disk emission models can describe the observed broad-line shapes well. 

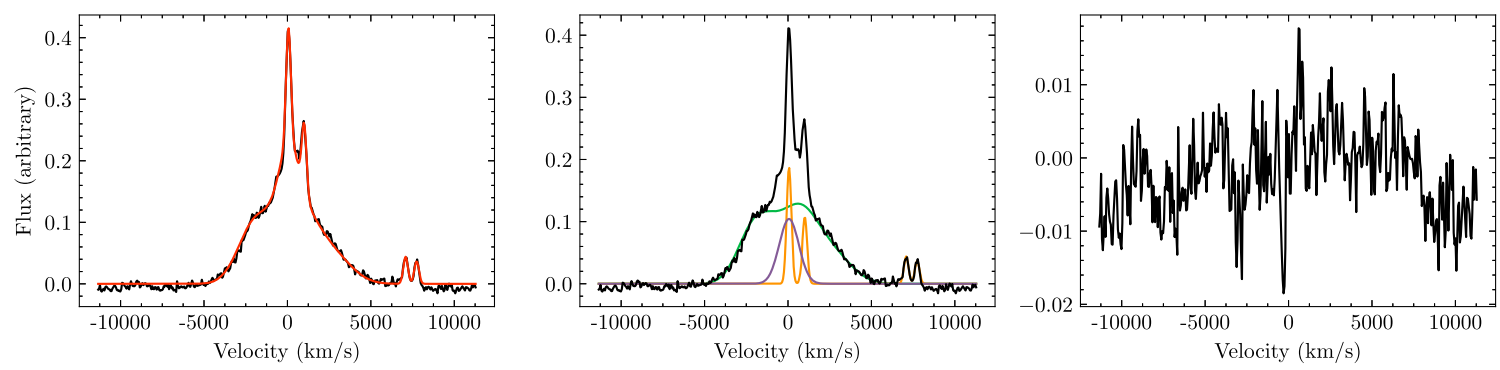

ZTF19aautrth
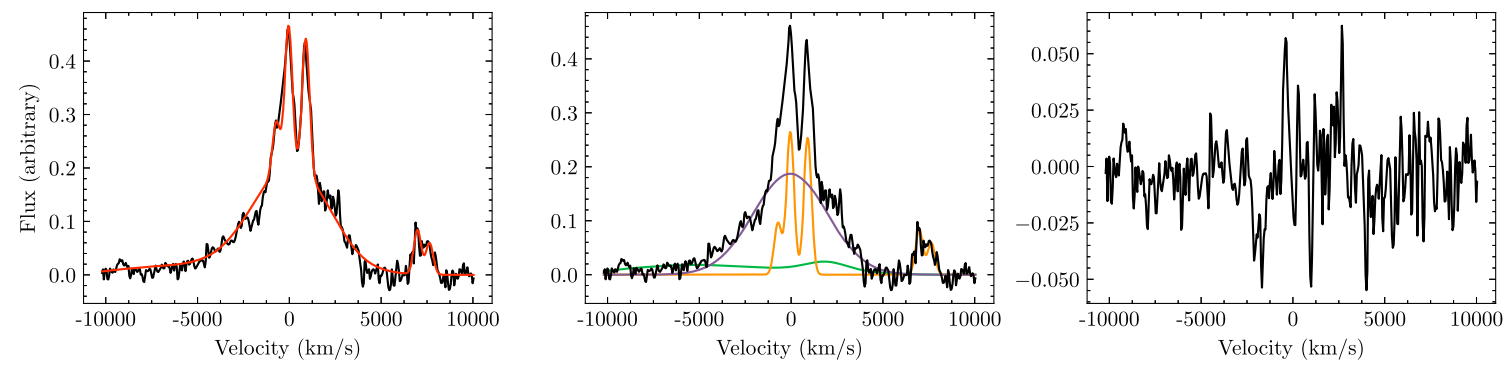

ZTF18aaxmrom
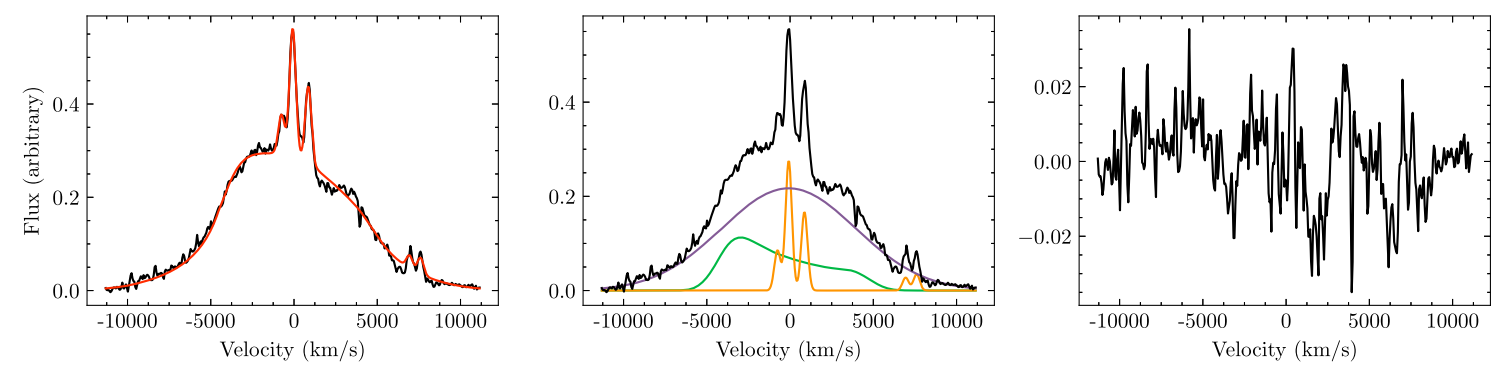

ZTF19aayrjsx
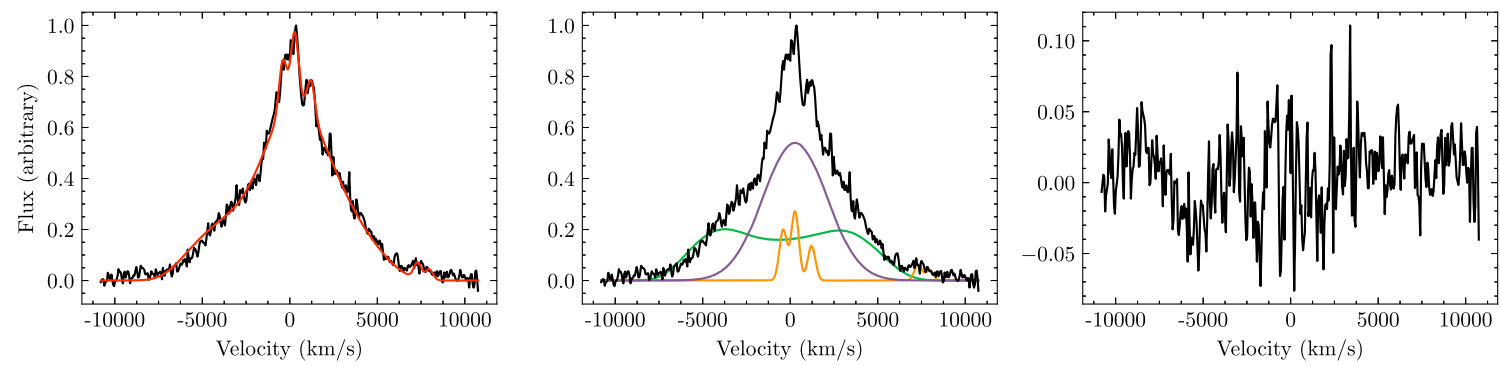

ZTF18aaoeobb
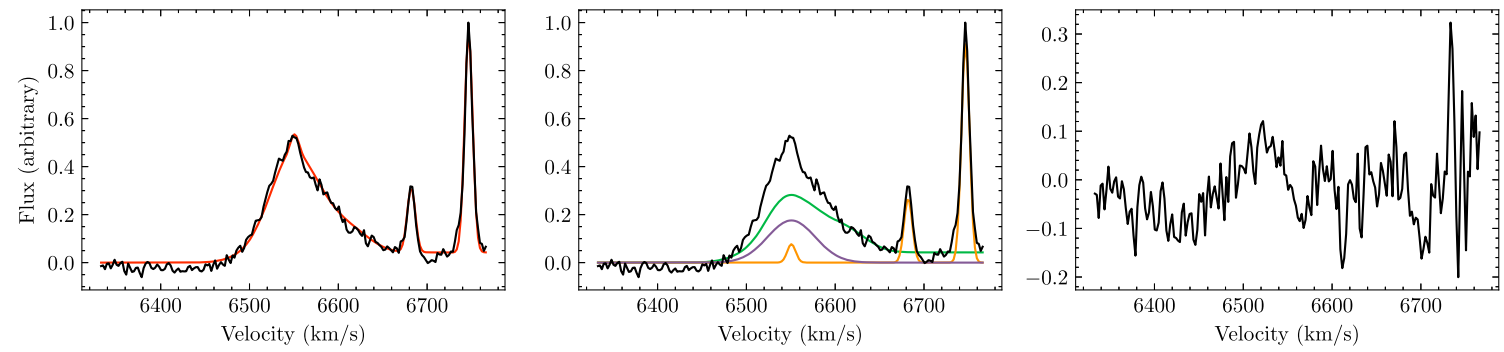

ZTF18aalsidi

Figure 13. Best-fit models of double-peaked $\mathrm{H} \alpha$ accretion disk emission in off-nuclear AGN candidates ZTF19aautrth, ZTF18aaxmrom, ZTF19aayrjsx, and ZTF18aaoeobb and $\mathrm{H} \beta$ disk emission in ZTF18aalsidi after subtracting a stellar continuum model derived from pPXF. On the left we show the continuum-subtracted data in black and the best-fit narrow emission line and accretion disk model in red. The middle plots show the separate narrow-line (green), accretion disk (orange), and central broad-line (purple) components of the fit. The rightmost plots show the error-weighted flux residuals after subtracting the best-fit model. 


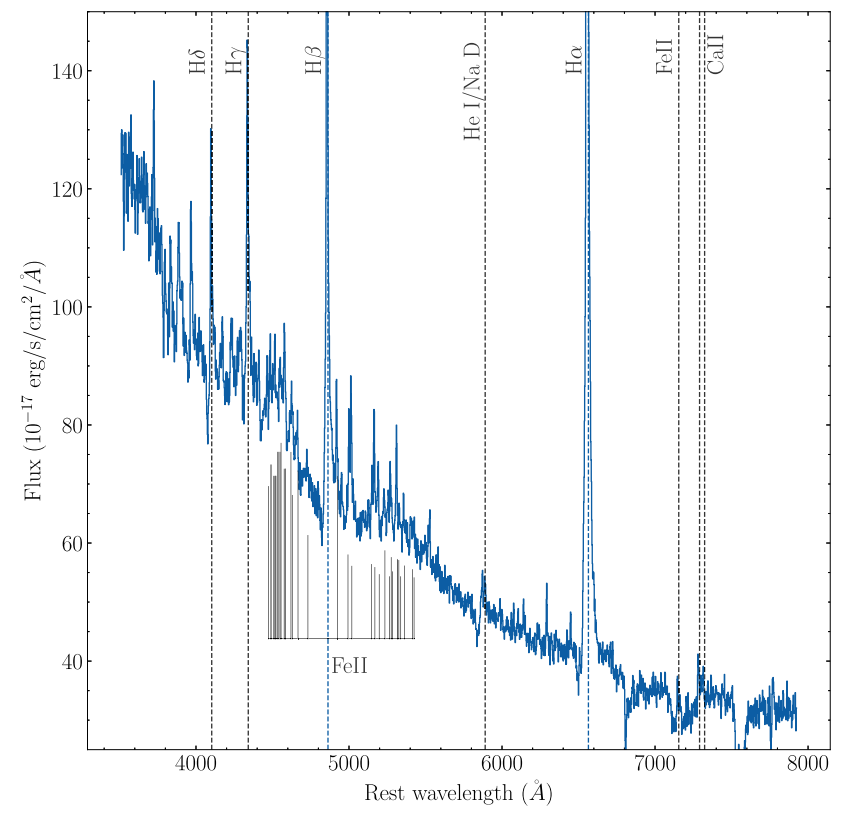

Complete spectrum
$\mathrm{H} \alpha$ broad line region.

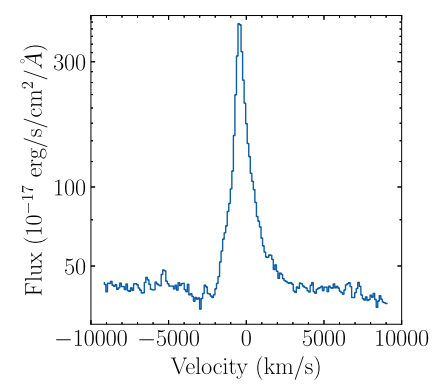

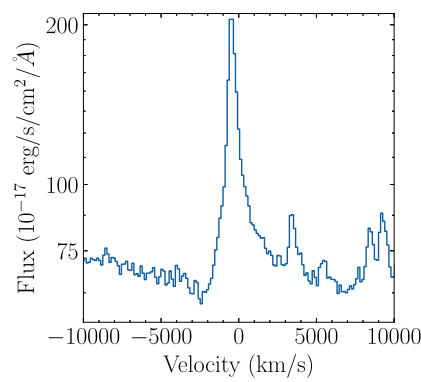

$\mathrm{H} \beta$ broad line region.
Figure 14. Spectrum of the flare ZTF19aafmjfw from SDSS 1133 taken on 2019 May 29 with the DeVeny spectrograph on the LDT. Subplot (a) shows the complete spectrum, while (b) and (c) show close-ups of $\mathrm{H} \alpha$ and $\mathrm{H} \beta$ in $\log$ scale to emphasize the P Cygni profile structure.

AGN, it may be induced by a recoil event. We therefore cannot yet completely rule out the recoil hypothesis in favor of an LBV or supernova impostor explanation.

\section{Conclusions}

We have described a novel search strategy for the discovery of spatially offset AGNs from recoiling SMBHs and ongoing galaxy mergers. This strategy uses ZTF difference imaging to find variable AGNs and Tractor forward modeling to determine the AGN position across multiple ZTF epochs and in deep, high-resolution Legacy Survey images.

We have found a sample of 52 AGNs in galaxy mergers and a sample of nine AGNs that may be spatially offset from their host galaxies. Five of the eight offset AGNs for which we have spectra of the $\mathrm{H} \alpha$ or $\mathrm{H} \beta$ BLRs show irregularly shaped broad Balmer lines with velocity offset broad peaks. These structures may arise due to emission from an unobscured, relativistic Keplerian accretion disk around the AGN. The $63 \%$ fraction of double-peaked emitters in the offset AGN sample is much larger than the $16 \%$ observed for the whole ZTF AGN sample.
The remaining three offset AGNs with spectra have Gaussian broad lines with velocities ranging in magnitude from 200 to $600 \mathrm{~km} \mathrm{~s}^{-1}$ relative to the narrow emission lines.

Our search strategy detected the variable object and recoiling SMBH candidate SDSS 1133 (Koss et al. 2014) when it rebrightened by $3 \mathrm{mag}$ in ZTF. Follow-up spectra showed the return of a blue continuum, blueshifted absorption lines, and [Fe II] $\lambda 7155$ and [Ca II] $\lambda \lambda 7291,7324$ forbidden lines, suggesting that the source may be either an LBV star that continues to show nonterminal outbursts or a recoiling AGN that continues to show variability.

Further multiwavelength follow-up is required to confirm that our recoiling SMBH candidates are not AGNs with undermassive hosts in mergers or chance coincidences with disturbed background galaxies. If even just a subset of our offset AGN candidates are confirmed to be recoiling black holes, their large spatial offsets could show that SMBH binaries with misaligned spins are able to form. Such binaries may be detectable at a later stage of evolution by the Laser Interferometer Space Antenna and would provide strong constraints on models of SMBH binary formation. The success of our variability-based search strategy with ZTF suggests that future searches for offset AGNs with the Vera Rubin Observatory (Ivezić et al. 2019) may yield large populations of recoiling SMBH candidates and AGNs in mergers.

We thank the anonymous reviewer for their helpful feedback. S.G. is supported in part by National Science Foundation grant 1616566. This work relied on observations obtained with the Samuel Oschin Telescope 48 inch and the 60 inch Telescope at the Palomar Observatory as part of the ZTF survey. ZTF is supported by the National Science Foundation under grant No. AST-1440341 and by a collaboration including Caltech, IPAC, the Weizmann Institute of Science, the Oskar Klein Centre at Stockholm University, the University of Maryland, the University of Washington, Deutsches Elektronen-Synchrotron and Humboldt University, Los Alamos National Laboratory, the TANGO Consortium of Taiwan, the University of Wisconsin at Milwaukee, and Lawrence Berkeley National Laboratory. Operations are conducted by COO, IPAC, and UW. The SED Machine is based upon work supported by the National Science Foundation under grant No. 1106171. This work was supported by the GROWTH project funded by the National Science Foundation under grant No. 1545949. M.M.K. acknowledges generous support from the David and Lucile Packard Foundation.

This research used resources of the National Energy Research Scientific Computing Center, a user facility supported by the Office of Science of the US Department of Energy (DOE) under contract No. DE-AC02-05CH11231. P.E.N. acknowledges support from the DOE under grant DE-AC0205CH11231, Analytical Modeling for Extreme-scale Computing Environments.

These results made use of the LDT at Lowell Observatory. Lowell is a private, nonprofit institution dedicated to astrophysical research and public appreciation of astronomy and operates the LDT in partnership with Boston University, the University of Maryland, the University of Toledo, Northern Arizona University, and Yale University. The upgrade of the DeVeny optical spectrograph has been funded by a generous grant from John and Ginger Giovale. 
This project used data obtained with the DECam, which was constructed by the Dark Energy Survey (DES) collaboration. Funding for the DES projects has been provided by the DOE, the US National Science Foundation, the Ministry of Science and Education of Spain, the Science and Technology Facilities Council of the United Kingdom, the Higher Education Funding Council for England, the National Center for Supercomputing Applications at the University of Illinois at Urbana-Champaign, the Kavli Institute of Cosmological Physics at the University of Chicago, the Center for Cosmology and AstroParticle Physics at the Ohio State University, the Mitchell Institute for Fundamental Physics and Astronomy at Texas A\&M University, Financiadora de Estudos e Projetos, Fundacao Carlos Chagas Filho de Amparo, Financiadora de Estudos e Projetos, Fundacao Carlos Chagas Filho de Amparo a Pesquisa do Estado do Rio de Janeiro, Conselho Nacional de Desenvolvimento Cientifico e Tecnologico and Ministerio da Ciencia, Tecnologia e Inovacao, Deutsche Forschungsgemeinschaft, and the collaborating institutions in the DES. The collaborating institutions are Argonne National Laboratory, the University of California at Santa Cruz, the University of Cambridge, Centro de Investigaciones Energeticas, Medioambientales y Tecnologicas-Madrid, the University of Chicago, University College London, the DES-Brazil Consortium, the University of Edinburgh, Eidgenossische Technische Hochschule Zurich, Fermi National Accelerator Laboratory, the University of Illinois at Urbana-Champaign, Institut de Ciencies de l'Espai (IEEC/CSIC), Institut de Fisica d'Altes Energies, Lawrence
Berkeley National Laboratory, Ludwig-Maximilians Universitat Munchen and the associated Excellence Cluster Universe, the University of Michigan, the National Optical Astronomy Observatory, the University of Nottingham, the Ohio State University, the University of Pennsylvania, the University of Portsmouth, SLAC National Accelerator Laboratory, Stanford University, the University of Sussex, and Texas A\&M University.

Software: Ampel (Nordin et al. 2019), Astropy (Robitaille et al. 2013; Price-Whelan et al. 2018), catsHTM (Soumagnac \& Ofek 2018), extcats (github.com/MatteoGiomi/extcats), GROWTH Marshal (Kasliwal et al. 2019), The Tractor (Lang et al. 2016).

\section{Appendix}

In this Appendix we show the distribution of magnitudeweighted AGN-host galaxy offset distributions derived from ZTF alert packet information about the transient and host positions for the sample of 3110 AGN which also underwent Tractor modeling. We also show the Rayleigh + exponential models used to derive probabilities that offsets arise from the exponential model component are therefore real. Figures 15 and 16 and show the r-band and g-band offset and probability distributions, respectively. Table 6 summarizes the archival ROSAT and X-ray detections and upper limits for the sample of $52 \mathrm{AGN}$ in galaxy mergers. 


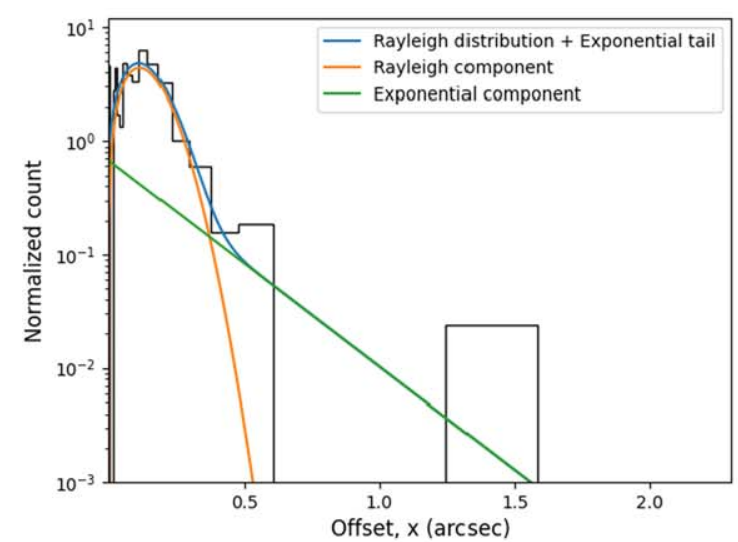

(a) AGN with a peak difference magnitude between 15 and 18 .

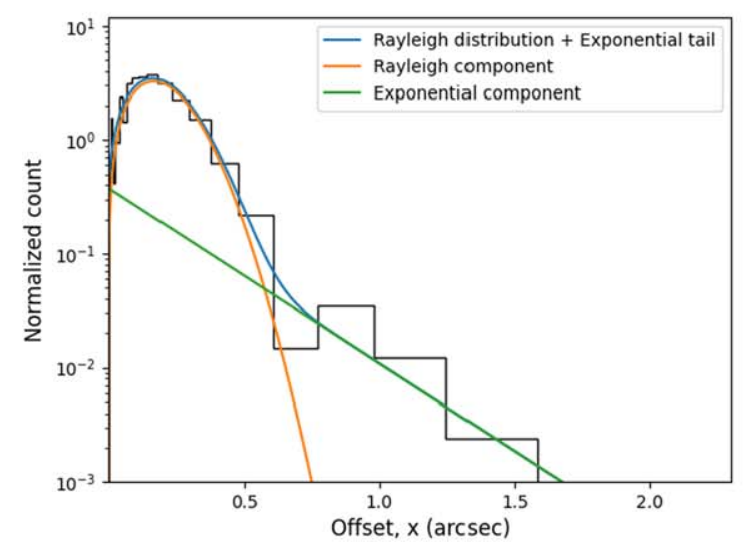

(c) AGN with a peak difference magnitude between 18 and 19.5 .

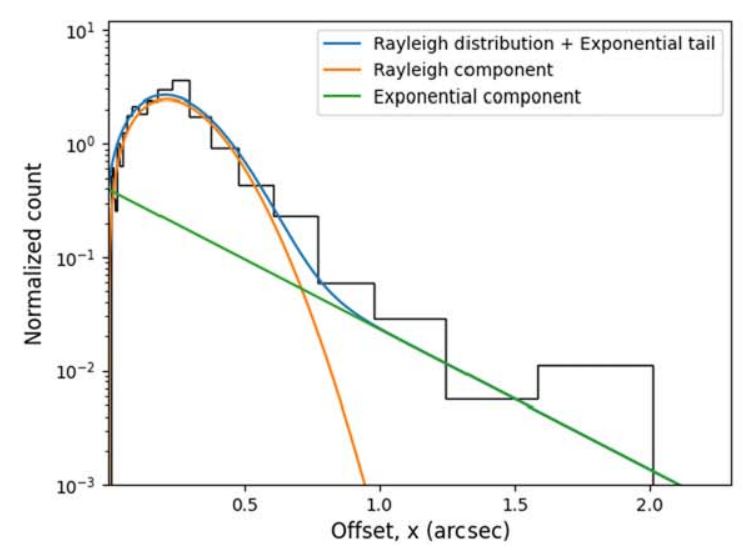

(e) AGN with a peak difference magnitude between 19.5 and 23.

Figure 15. Left: Normalized histogram with logarithmic bins for magnitude-weighted AGN-host offsets obtained from $r$-band ZTF difference images. The best-fit model of a mixture distribution with Rayleigh and exponential components is shown. Right: Probability that an offset greater than $R$ is drawn from the Rayleigh component of the mixture distribution shown in (a) instead of the exponential component. The offset where this probability is $0.3 \%$ is shown with a dashed line.

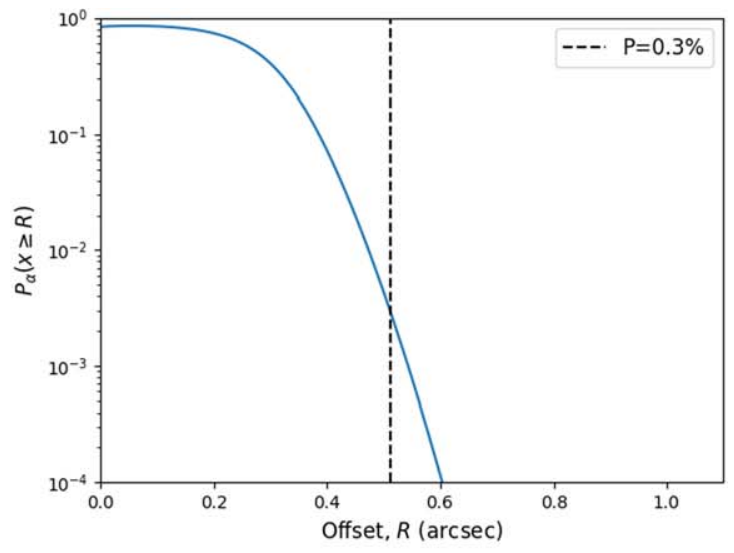

(b) $3 \sigma$ offset $=0.551 "$.

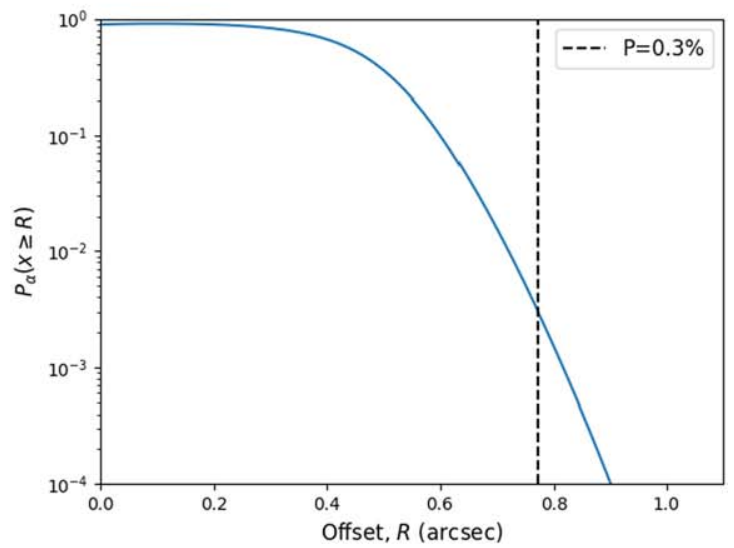

(d) $3 \sigma$ offset $=0.773 "$.

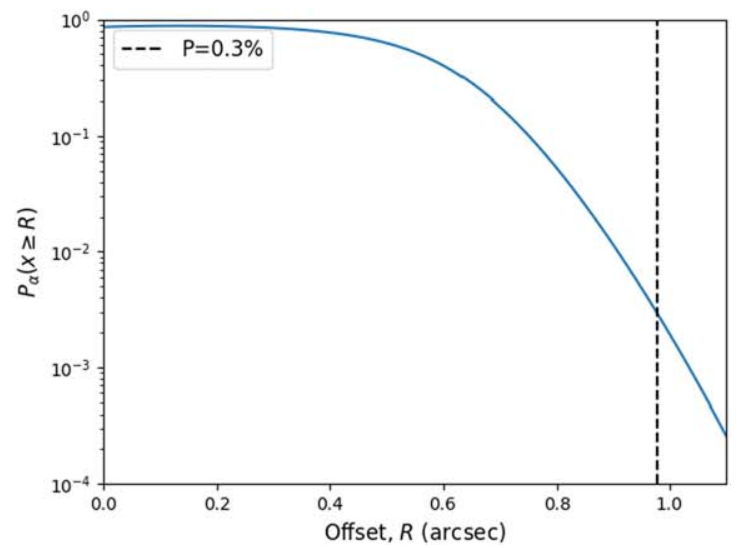

(f) $3 \sigma$ offset $=0.976 "$. 


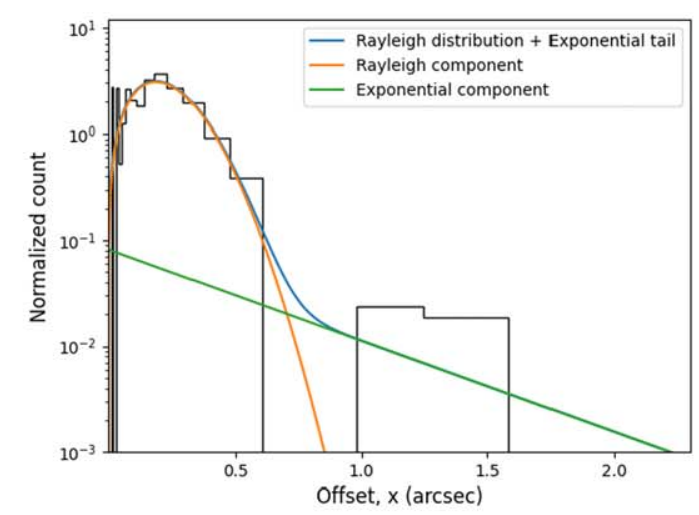

(a) AGN with a peak difference magnitude between 15 and 18 .

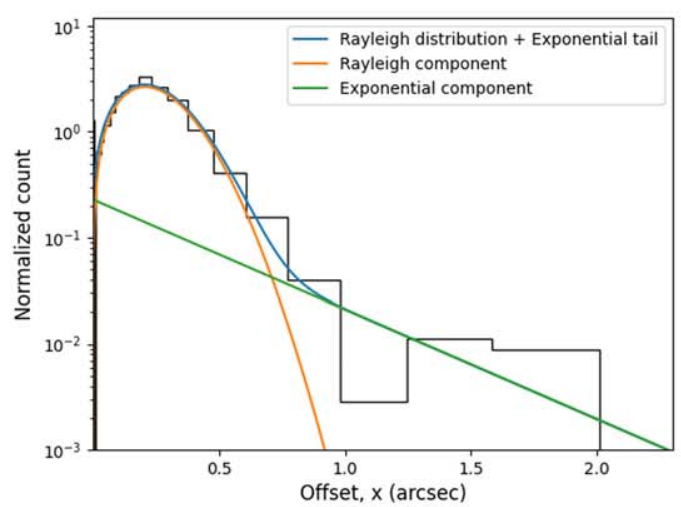

(c) AGN with a peak difference magnitude between 18 and 19.5

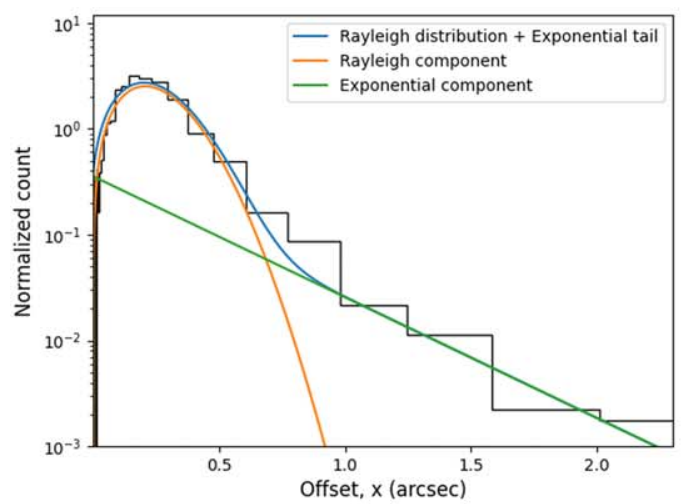

(e) AGN with a peak difference magnitude between 19.5 and 23.

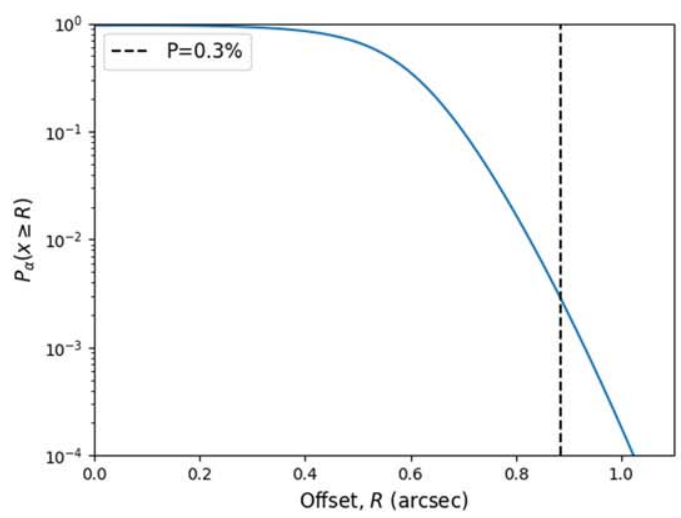

(b) $3 \sigma$ offset $=0.959 "$.

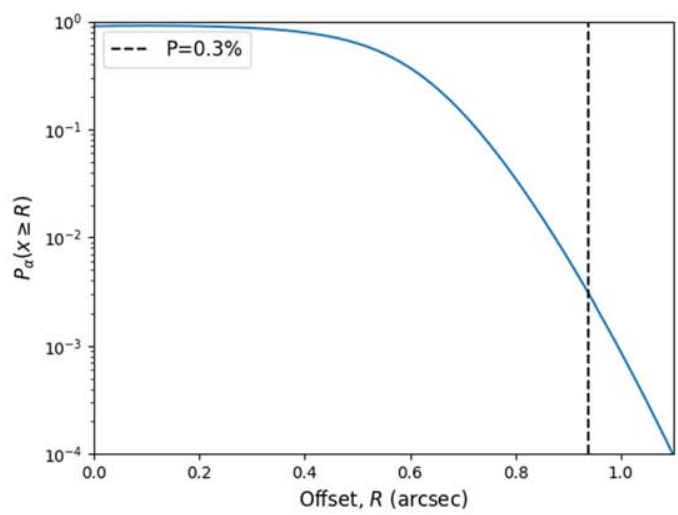

(d) $3 \sigma$ offset $=1.009 "$.

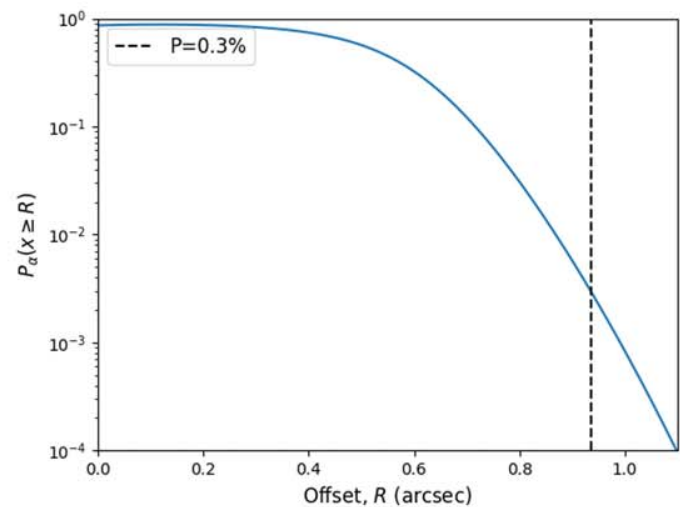

(f) $3 \sigma$ offset $=0.946 "$.

Figure 16. Left: Normalized histogram with logarithmic bins for magnitude-weighted AGN-host offsets obtained from $g$-band ZTF difference images. The best-fit model of a mixture distribution with Rayleigh and exponential components is shown. Right: Probability that an offset greater than $R$ is drawn from the Rayleigh component of the mixture distribution shown in (a) instead of the exponential component. The offset where this probability is $0.3 \%$ is shown with a dashed line. 
Table 6

Galaxy Merger X-Ray and Radio Detections

\begin{tabular}{|c|c|c|c|c|}
\hline ZTF Name & $\begin{array}{l}\text { ROSAT Flux } \\
\quad\left(\operatorname{cts~s}^{-1}\right)\end{array}$ & $\begin{aligned} & L_{2-10 \mathrm{keV}} \\
&\left(\times 10^{44} \mathrm{erg} \mathrm{s}^{-1}\right)\end{aligned}$ & $\begin{array}{c}\text { FIRST Flux } \\
\left(\times 10^{24} \mathrm{erg} \mathrm{cm}^{-2} \mathrm{~s}^{-1}\right)\end{array}$ & $\begin{array}{c}L_{5} \mathrm{GHz} \\
\left(\times 10^{55} \mathrm{erg} \mathrm{s}^{-1}\right)\end{array}$ \\
\hline ZTF18aaxvmpg & $0.196 \pm 0.022$ & $1.126 \pm 0.129$ & $<1.39$ & $<18.552$ \\
\hline ZTF18abamzru & $<0.05$ & $\ldots$ & $<1.33$ & $\ldots$ \\
\hline ZTF18aasxvyo & $1.728 \pm 0.06$ & $\ldots$ & $24.3 \pm 1.36$ & $\ldots$ \\
\hline ZTF18aaieguy & $<0.05$ & $\ldots$ & $<1.54$ & $<20.944$ \\
\hline ZTF19aakjemw & $0.031 \pm 0.011$ & $0.149 \pm 0.054$ & $<1.46$ & $<8.666$ \\
\hline ZTF18aaifbku & $0.302 \pm 0.025$ & $0.245 \pm 0.02$ & $185.3 \pm 1.5$ & $208.297 \pm 1.686$ \\
\hline ZTF18aampabj & $<0.05$ & $\ldots$ & $24.1 \pm 1.34$ & $335.637 \pm 18.662$ \\
\hline ZTF19aaagygp & $0.04 \pm 0.012$ & $\ldots$ & $\ldots$ & $\ldots$ \\
\hline ZTF18abujubn & $<0.05$ & $\ldots$ & $\ldots$ & $\ldots$ \\
\hline ZTF18acegbsb & $0.034 \pm 0.014$ & $0.009 \pm 0.004$ & $12.8 \pm 0.97$ & $4.206 \pm 0.319$ \\
\hline ZTF19aaozpdm & $<0.05$ & $\ldots$ & $<1.39$ & $\ldots$ \\
\hline ZTF18aacjltc & $0.051 \pm 0.015$ & $0.529 \pm 0.159$ & $<1.76$ & $<18.156$ \\
\hline ZTF18abtpite & $0.024 \pm 0.01$ & $\ldots$ & $\ldots$ & $\ldots$ \\
\hline ZTF18abvwrxu & $0.163 \pm 0.03$ & $\ldots$ & $<1.42$ & $\ldots$ \\
\hline ZTF19abaktpb & $<0.05$ & $\ldots$ & $\ldots$ & $\ldots$ \\
\hline ZTF18aaqjcxl & $<0.05$ & $\ldots$ & $<1.47$ & $<3.706$ \\
\hline ZTF18abyoivl & $<0.05$ & $\ldots$ & $<1.1$ & $\ldots$ \\
\hline ZTF18aabdiug & $<0.05$ & $\ldots$ & $37.4 \pm 1.43$ & $35.732 \pm 1.366$ \\
\hline ZTF19aaviuyv & $0.051 \pm 0.01$ & $\ldots$ & $\ldots$ & $\ldots$ \\
\hline ZTF18aabxczq & $<0.05$ & $\ldots$ & $<1.45$ & $<1.43$ \\
\hline ZTF18acvwlrf & $<0.05$ & $\ldots$ & $11.6 \pm 1.51$ & $192.822 \pm 25.1$ \\
\hline ZTF19aasejqv & $0.086 \pm 0.016$ & $0.721 \pm 0.135$ & $<1.34$ & $<22.161$ \\
\hline ZTF18aazogyo & $<0.05$ & $\ldots$ & $<1.44$ & $<2.41$ \\
\hline ZTF18aceypvy & $0.047 \pm 0.012$ & $0.215 \pm 0.055$ & $<1.39$ & $<10.349$ \\
\hline ZTF18acbweyd & $<0.05$ & $\ldots$ & $<1.36$ & $<14.168$ \\
\hline ZTF18acablce & $0.022 \pm 0.005$ & $\ldots$ & $\ldots$ & $\ldots$ \\
\hline ZTF18abhpvvr & $0.072 \pm 0.014$ & $\ldots$ & $\ldots$ & $\ldots$ \\
\hline ZTF19abfqmjg & $<0.05$ & $\ldots$ & $\ldots$ & $\ldots$ \\
\hline ZTF18abmqwgr & $0.077 \pm 0.015$ & $\ldots$ & $\ldots$ & $\ldots$ \\
\hline ZTF19aadgbih & $0.092 \pm 0.016$ & $0.603 \pm 0.102$ & $24.9 \pm 1.5$ & $279.113 \pm 16.814$ \\
\hline ZTF19aalpfan & $<0.05$ & $\ldots$ & $<1.48$ & $<2.056$ \\
\hline ZTF18aawwfep & $<0.05$ & $\ldots$ & $<1.39$ & $<15.897$ \\
\hline ZTF19aavxims & $0.041 \pm 0.015$ & $\ldots$ & $<1.52$ & $\ldots$ \\
\hline ZTF19aaaplct & $0.165 \pm 0.025$ & $\ldots$ & $<1.48$ & $\ldots$ \\
\hline ZTF18aajnqqv & $0.02 \pm 0.009$ & $0.026 \pm 0.012$ & $36.5 \pm 1.47$ & $59.825 \pm 2.409$ \\
\hline ZTF18abszfur & $<0.05$ & $\ldots$ & $<1.5$ & $<41.386$ \\
\hline ZTF19abucbkt & $<0.05$ & $\ldots$ & $\ldots$ & $\ldots$ \\
\hline ZTF18adbhlyb & $0.036 \pm 0.013$ & $0.253 \pm 0.09$ & $<1.34$ & $<17.931$ \\
\hline ZTF18acxhoij & $0.086 \pm 0.016$ & $\ldots$ & $\ldots$ & $\ldots$ \\
\hline ZTF18acajwep & $<0.05$ & $\ldots$ & $\ldots$ & $\ldots$ \\
\hline ZTF19abipoqj & $<0.05$ & $\ldots$ & $<1.05$ & $\ldots$ \\
\hline ZTF19abpkoou & $0.043 \pm 0.016$ & $\ldots$ & $<1.08$ & $\ldots$ \\
\hline ZTF18abztovy & $<0.05$ & $\ldots$ & $<1.54$ & $\ldots$ \\
\hline ZTF18acsllgd & $0.031 \pm 0.012$ & $\ldots$ & $\ldots$ & $\ldots$ \\
\hline ZTF19aanxrki & $0.068 \pm 0.014$ & $0.237 \pm 0.049$ & $<1.44$ & $<4.96$ \\
\hline ZTF18aamfuhc & $0.341 \pm 0.033$ & $0.417 \pm 0.04$ & $<1.42$ & $<2.693$ \\
\hline ZTF18aadwvyr & $0.06 \pm 0.014$ & $0.252 \pm 0.059$ & $<1.02$ & $<4.353$ \\
\hline ZTF19abauzsd & $0.027 \pm 0.009$ & $0.643 \pm 0.206$ & $<1.5$ & $<39.366$ \\
\hline ZTF18abufbsq & $0.048 \pm 0.013$ & $\ldots$ & $21.5 \pm 1.39$ & $\ldots$ \\
\hline ZTF18abzuzrg & $0.028 \pm 0.009$ & $0.169 \pm 0.053$ & $<1.45$ & $<13.173$ \\
\hline ZTF18abtmcdb & $0.032 \pm 0.01$ & $\ldots$ & $<1.11$ & $\ldots$ \\
\hline ZTF18aauhnby & $0.1 \pm 0.018$ & $0.113 \pm 0.021$ & $<1.53$ & $<3.168$ \\
\hline
\end{tabular}

Notes. X-ray and radio properties of the 52 ZTF AGNs in galaxy mergers. We show the 2-10 keV flux in counts per second for the AGNs with detections in the second ROSAT All-sky Survey and conversions to luminosity for those with a spectroscopically confirmed redshift. We also show the $20 \mathrm{~cm}$ flux from the FIRST survey, including upper limits where available, and the corresponding luminosities. 


\section{ORCID iDs}

Charlotte Ward (1D https://orcid.org/0000-0002-4557-6682 Suvi Gezari (i) https://orcid.org/0000-0003-3703-5154 Sara Frederick (10) https://orcid.org/0000-0001-9676-730X Erica Hammerstein (ib https://orcid.org/0000-0002-5698-8703 Peter Nugent (iD https://orcid.org/0000-0002-3389-0586 Eric C. Bellm (i) https://orcid.org/0000-0001-8018-5348 Dmitry A. Duev (10 https://orcid.org/0000-0001-5060-8733 Matthew J. Graham (i) https://orcid.org/0000-0002-3168-0139 Mansi M. Kasliwal (iD https://orcid.org/0000-0002-5619-4938 Ashish A. Mahabal (iD https://orcid.org/0000-0003-2242-0244 Frank J. Masci (i) https://orcid.org/0000-0002-8532-9395 Ben Rusholme (iD https://orcid.org/0000-0001-7648-4142 Maayane T. Soumagnac (iD https://orcid.org/0000-00016753-1488

Lin Yan (i) https://orcid.org/0000-0003-1710-9339

\section{References}

Afanasiev, A. V., Chilingarian, I. V., Mieske, S., et al. 2018, MNRAS, 477, 4856

Ahn, C. P., Seth, A. C., Brok, M. d., et al. 2017, ApJ, 839, 72

Ahn, C. P., Seth, A. C., Cappellari, M., et al. 2018, ApJ, 858, 102

Allen, J. T., Schaefer, A. L., Scott, N., et al. 2015, MNRAS, 451, 2780

Assef, R. J., Stern, D., Kochanek, C. S., et al. 2013, ApJ, 772, 32

Baldassare, V. F., Geha, M., \& Greene, J. 2018, ApJ, 868, 152

Baldassare, V. F., Geha, M., \& Greene, J. 2020, ApJ, 896, 10

Barbary, K., rbiswas4, Goldstein, D., et al. 2016, sncosmo/sncosmo: v1.4.0, Zenodo, doi:10.5281/zenodo. 168220

Bellm, E. C., Kulkarni, S. R., Graham, M. J., et al. 2019, PASP, 131, 018002 Bellovary, J. M., Governato, F., Quinn, T. R., et al. 2010, ApJL, 721, 148

Berczik, P., Merritt, D., Spurzem, R., \& Bischof, H.-P. 2006, ApJL, 642, L21 Blecha, L., Cox, T. J., Loeb, A., \& Hernquist, L. 2011, MNRAS, 412, 2154 Blecha, L., \& Loeb, A. 2008, MNRAS, 390, 1311

Blecha, L., Sijacki, D., Kelley, L. Z., et al. 2016, MNRAS, 456, 961 Bogdanović, T., Reynolds, C. S., \& Miller, M. C. 2007, ApJL, 661, L147 Boles, T. 2009, CBET, 1931, 1

Boller, T., Freyberg, M. J., Trümper, J., et al. 2016, A\&A, 588, A103 Bonning, E. W., Shields, G. A., \& Salviander, S. 2007, ApJL, 666, L13 Brescia, M., Cavuoti, S., \& Longo, G. 2015, MNRAS, 450, 3893 Burke, C. J., Baldassare, V. F., Liu, X., et al. 2020, ApJL, 894, L5 Butler, N. R., \& Bloom, J. S. 2011, AJ, 141, 93

Campanelli, M., Lousto, C. O., Zlochower, Y., \& Merritt, D. 2007, PhRvL, 98, 8

Canalizo, G., \& Stockton, A. 2001, ApJ, 555, 719

Cappellari, M. 2017, MNRAS, 466, 798

Cappellari, M., \& Emsellem, E. 2004, PASP, 116, 138

Chambers, K. C., Magnier, E. A., Metcalfe, N., et al. 2016, arXiv:1612.05560

Chen, K., \& Halpern, J. P. 1989, ApJ, 344, 115

Chen, K., Halpern, J. P., \& Filippenko, A. V. 1989, ApJ, 339, 742

Chiaberge, M., Ely, J. C., Meyer, E. T., et al. 2017, A\&A, 600, A57

Chiaberge, M., Gilli, R., Lotz, M., J., \& Norman, C. 2015, ApJ, 806, 147

Chiaberge, M., Tremblay, G. R., Capetti, A., \& Norman, C. 2018, ApJ, 861, 56

Cid Fernandes, R., Stasińska, G., Mateus, A., \& Vale Asari, N. 2011, MNRAS, 413, 1687

Civano, F., Elvis, M., Lanzuisi, G., et al. 2010, ApJ, 717, 209

Comerford, J. M., Barrows, R. S., Greene, J. E., \& Pooley, D. 2017, ApJ, 847,41

Comerford, J. M., Gerke, B. F., Newman, J. A., et al. 2009, ApJ, 698, 956

Comerford, J. M., \& Greene, J. E. 2014, ApJ, 789, 112

Condon, J. J., Cotton, W. D., Greisen, E. W., et al. 1998, AJ, 115, 1693

Condon, J. J., Darling, J., Kovalev, Y. Y., \& Petrov, L. 2017, ApJ, 834, 184

Congiu, E., Berton, M., Giroletti, M., et al. 2017, A\&A, 603, A32

Dekany, R., Smith, R. M., Riddle, R., et al. 2020, PASP, 132, 038001

Dey, A., Schlegel, D. J., Lang, D., et al. 2019, AJ, 157, 168

Drake, A. J., Djorgovski, S. G., Mahabal, A., et al. 2009, ApJ, 696, 870

Duev, D. A., Mahabal, A., Masci, F. J., et al. 2019, MNRAS, 489, 3582 Eracleous, M., \& Halpern, J. P. 1994, ApJS, 90, 1

Escala, A., Larson, R. B., Coppi, P. S., \& Mardones, D. 2005, ApJ, 630, 152 Evans, I. N., Primini, F. A., Glotfelty, K. J., et al. 2010, ApJS, 189, 37

Farrell, S. A., Webb, N. A., Barret, D., Godet, O., \& Rodrigues, J. M. 2009, Natur, 460, 73
Ferrarese, L., \& Ford, H. 2005, SSRv, 116, 523

Filippenko, A. V. 1997, ARA\&A, 35, 309

Flesch, E. W. 2015, PASA, 32, e010

Foley, R. J., Berger, E., Fox, O., et al. 2011, ApJ, 732, 32

Fu, H., Myers, A. D., Djorgovski, S. G., \& Yan, L. 2011a, ApJ, 733, 103

Fu, H., Zhang, Z. Y., Assef, R. J., et al. 2011b, ApJL, 740, L1

Glikman, E., Simmons, B., Mailly, M., et al. 2015, ApJ, 806, 218

Graham, M. J., Kulkarni, S. R., Bellm, E. C., et al. 2019, PASP, 131, 078001

Halpern, J. P., Impey, C. D., Bothun, G. D., et al. 1986, ApJ, 302, 711

Helfand, D. J., White, R. L., \& Becker, R. H. 2015, ApJ, 801, 26

Hemberger, D. A., Lovelace, G., Loredo, T. J., et al. 2013, PhRvD, 88, 064014

Huang, Y., Liu, X. W., Yuan, H. B., et al. 2014, MNRAS, 439, 2927

Hwang, H.-C., Shen, Y., Zakamska, N., \& Liu, X. 2020, ApJ, 888, 73

Ivezić, v., Kahn, S. M., Tyson, J. A., et al. 2019, ApJ, 873, 111

Ivison, R. J., Smail, I., Amblard, A., et al. 2012, MNRAS, 425, 1320

Jarrett, T. H., Chester, T., Cutri, R., et al. 2000, AJ, 119, 2498

Jonker, P. G., Torres, M. A., Fabian, A. C., et al. 2010, MNRAS, 407, 645

Kalfountzou, E., Lleo, M. S., \& Trichas, M. 2017, ApJL, 851, L15

Kasliwal, M. M., Cannella, C., Bagdasaryan, A., et al. 2019, PASP, 131, 038003

Kauffmann, G., Heckman, T. M., Tremonti, C., et al. 2003, MNRAS, 346, 1055

Keel, W. C., Bennert, V. N., Pancoast, A., et al. 2019, MNRAS, 483, 4847

Kellermann, K. I., Sramek, R., Schmidt, M., Shaffer, D. B., \& Green, R. 1989, AJ, 98, 1195

Kim, D.-C., Yoon, I., Privon, G. C., et al. 2017, ApJ, 840, 71

Kocevski, D. D., Brightman, M., Nandra, K., et al. 2015, ApJ, 814, 104

Komossa, S., Burwitz, V., Hasinger, G., et al. 2003, ApJL, 582, L15

Kormendy, J., \& Richstone, D. 1995, ARA\&A, 33, 581

Koss, M., Blecha, L., Mushotzky, R., et al. 2014, MNRAS, 445, 515

Koss, M., Mushotzky, R., Treister, E., et al. 2011, ApJL, 735, L42

Koss, M., Mushotzky, R., Treister, E., et al. 2012, ApJL, 746, L22

Koss, M. J., Blecha, L., Bernhard, P., et al. 2018, Natur, 563, 214

Lang, D., Hogg, D. W., \& Mykytyn, D. 2016, The Tractor: Probabilistic Astronomical Source Detection and Measurement, Astrophysics Source Code Library, ascl:1604.008

Lena, D., Robinson, A., Marconi, A., et al. 2014, ApJ, 795, 146

Liu, X., Guo, H., Shen, Y., Greene, J. E., \& Strauss, M. A. 2018, ApJ, 862, 29

Liu, X., Shen, Y., Strauss, M. A., \& Greene, J. E. 2010, ApJ, 708, 427

Liu, X., Shen, Y., Strauss, M. A., \& Hao, L. 2011, ApJ, 737, 101

Lodato, G., \& Facchini, S. 2013, MNRAS, 433, 2157

Loeb, A. 2007, PhRvL, 99, 41103

Mahabal, A., Rebbapragada, U., Walters, R., et al. 2019, PASP, 131, 038002

Masci, F. J., Laher, R. R., Rusholme, B., et al. 2019, PASP, 131, 018003

Maza, J., Hamuy, M., Antezana, R., et al. 2009, CBET, 1928, 1

McLure, R. J., \& Dunlop, J. S. 2002, MNRAS, 331, 795

Mezcua, M., \& Domínguez Sánchez, H. 2020, ApJL, 898, L30

Miller, A. A., Kulkarni, M. K., Cao, Y., et al. 2017, AJ, 153, 73

Milosavljević, M., \& Merritt, D. 2001, ApJ, 563, 34

Nordin, J., Brinnel, V., van Santen, J., et al. 2019, A\&A, 631, A147

Pastorello, A., Reguitti, A., Morales-Garoffolo, A., et al. 2019, A\&A, 628, A93

Patterson, M. T., Bellm, E. C., Rusholme, B., et al. 2019, PASP, 131, 18001

Phillips, M. M. 1976, ApJ, 208, 37

Plotkin, R. M., Anderson, S. F., Hall, P. B., et al. 2008, AJ, 135, 2453

Price-Whelan, A. M., Sipőcz, B. M., Günther, H. M., et al. 2018, AJ, 156, 123

Reines, A. E., \& Comastri, A. 2016, PASA, 33, e054

Reines, A. E., Condon, J. J., Darling, J., \& Greene, J. E. 2020, ApJ, 888, 36 Robinson, A., Young, S., Axon, D. J., Kharb, P., \& Smith, J. E. 2010, ApJL, 717,122

Robitaille, T. P., Tollerud, E. J., Greenfield, P., et al. 2013, A\&A, 558, A33 Rots, A. H., \& Budavári, T. 2011, ApJS, 192, 8

Schnittman, J. D. 2013, CQGra, 30, 244007

Seth, A. C., Van Den Bosch, R., Mieske, S., et al. 2014, Natur, 513, 398

Shabala, S. S., Deller, A., Kaviraj, S., et al. 2017, MNRAS, 464, 4706

Shen, Y., Hwang, H.-c., Zakamska, N., \& Liu, X. 2019, ApJL, 885, L4

Shields, G. A., Bonning, E. W., \& Salviander, S. 2009a, ApJ, 696, 1367

Shields, G. A., Rosario, D. J., Smith, K. L., et al. 2009b, ApJ, 707, 936

Skipper, C. J., \& Browne, I. W. A. 2018, MNRAS, 475, 5179

Smith, K. L., Shields, G. A., Bonning, E. W., et al. 2010, ApJ, 716, 866

Solovyeva, Y., Vinokurov, A., Fabrika, S., et al. 2019, MNRAS: Lett., 484, L24

Soumagnac, M. T., \& Ofek, E. O. 2018, PASP, 130, 075002

Steinhardt, C. L., Schramm, M., Silverman, J. D., et al. 2012, ApJ, 759, 24

Strateva, I. V., Strauss, M. A., Hao, L., et al. 2003, AJ, 126, 1720

Surace, J. A., Sanders, D. B., Vacca, W. D., Veilleux, S., \& Mazzarella, J. M. 1998, ApJ, 492, 116 
Tachibana, Y., \& Miller, A. A. 2018, PASP, 130, 128001

Treister, E., Schawinski, K., Urry, C. M., \& Simmons, B. D. 2012, ApJL, 758, L39

van Velzen, S., Gezari, S., Cenko, S. B., et al. 2019, ApJ, 872, 198

van Velzen, S., Gezari, S., Hammerstein, E., et al. 2021, ApJ, 908, 4

Van Wassenhove, S., Volonteri, M., Mayer, L., et al. 2012, ApJL, 748, 5

Voggel, K. T., Seth, A. C., Baumgardt, H., et al. 2019, ApJ, 871, 159
Volonteri, M. 2007, ApJL, 663, L5

Volonteri, M., Gültekin, K., \& Dotti, M. 2010, MNRAS, 404, 2143

Volonteri, M., \& Madau, P. 2008, ApJL, 687, L57

Volonteri, M., \& Natarajan, P. 2009, MNRAS, 400, 1911

Volonteri, M., \& Perna, R. 2005, MNRAS, 358, 913

Wang, J. M., Chen, Y. M., Hu, C., et al. 2009, ApJ, 705, 76

Xu, D., \& Komossa, S. 2009, ApJ, 705, 20 